Article

\title{
Intelligent Deep Models Based on Scalograms of Electrocardiogram Signals for Biometrics
}

\author{
Yeong-Hyeon Byeon, Sung-Bum Pan $(1)$ and Keun-Chang Kwak *(i) \\ Department of Control and Instrumentation Engineering, Chosun University, Gwangju 61452, Korea; \\ qasdfghjt@hanmail.net (Y.-H.B.); sbpan@chosun.ac.kr (S.-B.P.) \\ * Correspondence: kwak@chosun.ac.kr; Tel.: +82-062-230-6086
}

Received: 5 January 2019; Accepted: 20 February 2019; Published: 22 February 2019

\begin{abstract}
This paper conducts a comparative analysis of deep models in biometrics using scalogram of electrocardiogram (ECG). A scalogram is the absolute value of the continuous wavelet transform coefficients of a signal. Since biometrics using ECG signals are sensitive to noise, studies have been conducted by transforming signals into a frequency domain that is efficient for analyzing noisy signals. By transforming the signal from the time domain to the frequency domain using the wavelet, the 1-D signal becomes a 2-D matrix, and it could be analyzed at multiresolution. However, this process makes signal analysis morphologically complex. This means that existing simple classifiers could perform poorly. We investigate the possibility of using the scalogram of ECG as input to deep convolutional neural networks of deep learning, which exhibit optimal performance for the classification of morphological imagery. When training data is small or hardware is insufficient for training, transfer learning can be used with pretrained deep models to reduce learning time, and classify it well enough. In this paper, AlexNet, GoogLeNet, and ResNet are considered as deep models of convolutional neural network. The experiments are performed on two databases for performance evaluation. Physikalisch-Technische Bundesanstalt (PTB)-ECG is a well-known database, while Chosun University (CU)-ECG is directly built for this study using the developed ECG sensor. The ResNet was $0.73 \%-0.27 \%$ higher than AlexNet or GoogLeNet on PTB-ECG—and the ResNet was $0.94 \%-0.12 \%$ higher than AlexNet or GoogLeNet on CU-ECG.
\end{abstract}

Keywords: biometric; deep learning; electrocardiogram; comparative analysis; scalogram

\section{Introduction}

Biometrics is a technology that automatically determines the identity of people using their physical and behavioral characteristics. Smartphones and IoT (Internet of Things) devices have become widespread, and the performance of their firmware has improved, so that they have been able to cover high computational load by themselves. Moreover, by receiving only results against the request for complicated analysis from a server through the Internet, various services can be implemented. If a service provider responds to requests indiscriminately, it may be exploited for crime, so it should be limited to the authorized person, and the required technology is authentication. Authentication methods of security-dependent services, such as access to private clouds and payments using smartphone banking, are moving toward applying convenient biometrics [1-6].

The characteristics used in biometric study include face [7], iris [8], gait [9], sweat pore [10], finger-vein [11], palmprint, palmvein [12], voice [13], handwritten signature [14], and electrocardiogram (ECG) [15]. The disadvantages of each characteristic include the face having restrictions on glasses, mask, and makeup, and the original face for recognition should be recovered. It is likely to be exploited for crime because it is vulnerable to camouflage. The iris has to be put close to a camera to be captured, and some glasses on faces are constrained for recognition. In order 
to measure the iris well, the eyes should be wide open, and the shape of iris varies according to the focus of the eyes. Using sweat pore, fingerprint, or palmvein as characteristics can be uncomfortable depending on the situation, because the hand cannot be used while measuring the data. In the case of gait, a person must perform a series of actions according to the scenario to obtain data. The voice is difficult to use in a noisy environment, and there is the inconvenience that a person must intentionally vocalize. The handwritten signature needs a pen to enter data, the hand is busy during writing, and the hand-written signature can be easily intentionally copied. The electrocardiogram is sensitive to the state and noise of the measuring signal [7-15].

The electrocardiogram is a measure of the change in voltage during the cardiac cycle. This change has uniqueness, because each person has a physically different body shape [16]. Recently, biometrics using the electrocardiogram has been carried out, and has shown good performance [17-19]. The complexity of the signal measurement process has been simplified, and measuring devices have been evolving into small, lightweight products in wearable form. Because the electrocardiogram signal is linked to the health check function, it is highly utilized. It is also advantageous for security, because the heart-generated signal is concealed inside the body. Through the sensor of the individual device, it is possible to measure without constraint anytime and anywhere. Biometrics using the electrocardiogram is sensitive to the state of the body and noise at measure, but studies are being conducted to solve these problems [16].

Since biometrics using electrocardiogram signals are sensitive to noise, studies have been conducted by converting signals into the frequency domain. Commonly used methods for the frequency analysis of signals are discrete cosine transform (DCT), continuous wavelet transform (CWT), and discrete wavelet transform (DWT). Li and Zhou performed electrocardiogram classification using wavelet packet entropy and random forests [20]. DCT is a linear combination of cosine functions with different frequencies that represent a signal and can compress the signal. Khorrami and Moavenian classified the electrocardiogram using DCT coefficients as a feature. This was compared with the classification results of features using CWT and DWT [21]. Song et al. used wavelet transform to extract 17 features from electrocardiogram signals, and features were used after dimensional reduction through linear discriminant analysis (LDA) [22]. Yu and Chen used some statistical features after decomposing the signal into sub-bands, using two-level DWT [23]. Ye et al. applied principal component analysis (PCA) to RR and features extracted from electrocardiogram by DWT and ICA (independent component analysis) [20,24]. Normally, the results of those transforms are 2-dimensional with axes of time and frequency. As studies to try signal processing in two dimensions, Soualhi et al. changed the electrocardiogram into a synchronous representation, then rearranged it every period to construct a two-dimensional image [25]. Zhai and Tin constructed a dual beat coupling matrix with beat morphology and beat-to-beat correlation, using two adjacent electrocardiogram beats [26].

In recent years, deep learning has been developed, breaking many existing limitations, and still, further improved methods are being proposed. Deep learning [27] is a neural network in which various types of layers are added, and many layers are piled up starting from the existing multilayer perceptron [28] in machine learning, and the problems that could not be solved with a small number of layers are solved by deep layers. There are deep neural networks [29], convolutional neural networks (CNN) [30], recurrent neural networks [31], deep belief networks [32], deep Q-networks [33], and so forth. One of the most prominent methods in image processing is a convolutional neural network that is designed to automatically learn various filters for feature extraction, and automatically classify the feature vectors. In the past, feature extraction was originally designed by humans, and machine learning was used only for classification. However, the convolutional neural network automatically learns not only classifiers, but also good features [34]. There are deep models with good performance in the ILSVRC (ImageNet Large Scale Visual Recognition Challenge) using the deep convolutional neural network [35-37]. When training data is small or hardware is insufficient for training, transfer learning can be used with pretrained deep models to reduce learning time and classify it well enough [38]. AlexNet [35], GoogLeNet [36], and ResNet [37] are existing deep models. AlexNet, which is similar 
to the existing LeNet [39], consists of seven layers and it is paralleled using two graphics processing units (GPUs) [35]. GoogLeNet is made up of 22 layers, and nine inception modules are applied [36]. ResNet is made up of 152 layers, and is added with residual learning, which reduces the time that is required to learn deep network, and enables successful learning, although it is very deep [37].

By transforming signal from time domain to frequency domain using the wavelet, the 1-D signal becomes a 2-D matrix, and it can be analyzed on multiresolution. However, this process makes morphologically complex signal analysis. This means that existing simple classifiers could be poor in performance. We investigated the possibility of using the image of frequency as input to CNN of deep learning, which exhibits optimal performance for the classification of morphological imagery. The work that compares the performance of deep models with input of the 2-D matrix using wavelet transform from ECG signals has not been studied so far. Thus, we focus on develop the most representative popular models frequently used in conjunction with deep learning models to perform ECG-based biometrics. The frequency image is here termed scalogram. AlexNet, GoogLeNet, and ResNet are considered deep models of the convolutional neural network. The novelty lies in the usage of scalogram to represent ECG features and testing its strength in biometric classification. Testing this method of input features in the form of image is appreciated for biometrics as time series of ECG signal does not visibly represent any differences regarding biometric analysis. The experiments were performed on two databases for performance evaluation. Physikalisch-Technische Bundesanstalt (PTB)-ECG is a well-known database, while Chosun university (CU)-ECG is directly built for this study using the developed ECG sensor. The ResNet was $0.73 \%-0.27 \%$ higher than AlexNet or GoogLeNet on PTB-ECG—and the ResNet was $0.94 \%-0.12 \%$ higher than AlexNet or GoogLeNet on CU-ECG.

In this paper, we performed comparative analysis of deep models in biometrics using scalogram of electrocardiogram. Section 2 describes deep models of convolutional neural network, while Section 3 discusses the biometrics method using scalogram of electrocardiogram. Section 4 presents the experimental results, while Section 5 concludes the paper.

\section{Deep Models of Convolutional Neural Network}

\subsection{AlexNet}

AlexNet is a structure of deep learning applied in parallel to two GPUs, with five convolutional layers, three pooling layers, two fully connected layers, and an output layer. The input image must be converted to a size of $227 \times 227$. Figure 1 shows the structure of AlexNet. The input image is passed in parallel to calculate the convolution with 96 filters of $11 \times 11 \times 3$ and a 4-stride, so two feature cubes of $55 \times 55 \times 48$ are created. Next, ReLU (Rectified Linear Unit) of an active function and LRN (Local Response Normalization) are applied to the cubes. Max-pooling with filter of $3 \times 3 \times 1$ and 2 -stride for each cube reduces the dimension to $27 \times 27 \times 48$ each. The cubes become $27 \times 27 \times 128$ each using convolution with 256 filters of $5 \times 5 \times 48$, 1-stride, and 2-padding, and are applied by ReLU and LRN. The cubes become $13 \times 13 \times 128$ each by max-pooling with filter of $3 \times 3 \times 1$ and 2 -stride. Their concatenated cube of $13 \times 13 \times 256$ becomes two $13 \times 13 \times 192$ cubes in parallel by convolution with 192 filters of $3 \times 3 \times 256$, 1-stride, and 1-padding twice, and then ReLU is applied. The cubes become $13 \times 13 \times 192$ each by convolution with 192 filters of $3 \times 3 \times 192$, 1-stride, and 1-padding, and then ReLU is applied. The cubes become $13 \times 13 \times 128$ by convolution with 128 filters of $3 \times 3 \times 192$, 1-stride, and 1-padding. The cubes become $6 \times 6 \times 128$ by max-pooling with filter of $3 \times 3 \times 1$, 2-stride, then are reshaped to $4608 \times 1$ as a one-dimensional vector. This is mapped to 2048 nodes, and then ReLU and dropout are applied, and that is repeated once more. Then it maps to 1000 nodes, which means the number of classes, and then softmax is applied for classification [35]. 


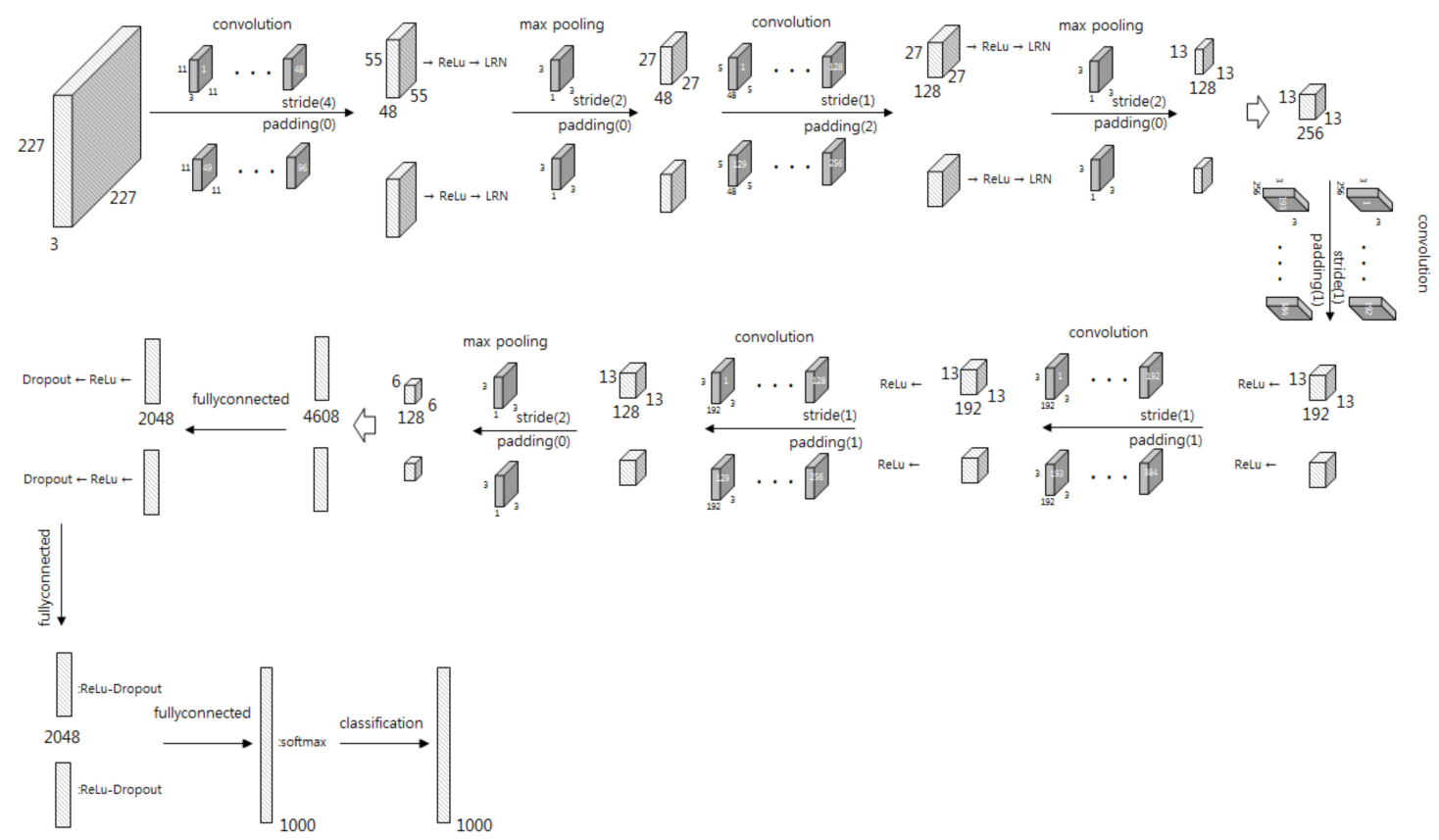

Figure 1. Structure of AlexNet.

\subsection{GoogLeNet}

GoogLeNet consists of nine inception layers. The idea of an inception layer is to keep the resolution good for small information, while covering a larger area in the image. Therefore, convolutions are performed at various sizes in parallel, from $1 \times 1$, which is the most accurate, to $5 \times 5$. Figure 2 shows the structure of the inception module. In inception, the convolution with filter of $1 \times 1$ reduces the number of feature maps, thereby reducing the amount of computation. Thus, the inception performs $1 \times 1$ convolutions first, and then performs convolutions of different sizes. In addition, max-pooling has been added to summarize the contents of the previous layer, and all the results are concatenated on the next layer. Also, two auxiliary classifiers were used in the middle of GoogLeNet structure, to avoid the vanishing gradient problem during learning [36].

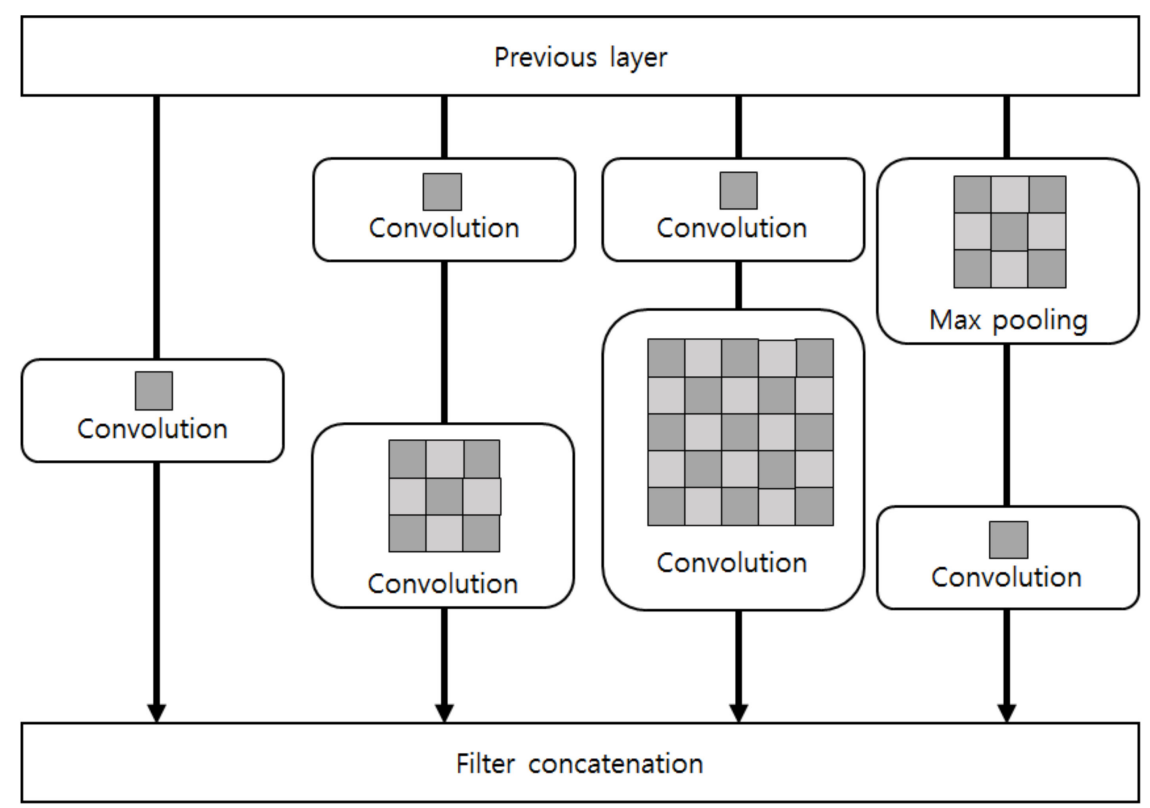

Figure 2. Structure of inception module. 


\subsection{ResNet}

As the layer of the neural network becomes deeper, the problems of vanishing gradient, exploding gradient, and degradation occur. The vanishing gradient means that the gradient of the propagation becomes too small, while the exploding gradient means that the gradient of the propagation becomes too big, which makes it fail at training. Degradation means that deep neural networks perform worse than shallow neural networks, although no overfitting has occurred. ResNet improves the learning efficiency by reusing the input features of the upper layer to solve this problem. Figure 3 shows the principle of residual learning. The input of $X$ makes the output of $Y$, and then the input of $X$ is added to the output of $\mathrm{Y}$ again. Then, learning is performed for $\operatorname{ReLU}(\mathrm{W} \times \mathrm{X})$ to converge on 0 , and this means that the output of $Y$ is almost the same value as $X$. This is not a problem even if the intermediate weights are small, and small changes in the input are also reflected in the output. The number of intermediate weight layers can be placed randomly, and ResNet is a model that is stacked deeply in this way. Its basic framework is referred to VGGNet [40], it uses convolution only with a filter of $3 \times 3$ and does not use pooling or dropout. To reduce the size of the feature map, it uses convolution with 2-stride instead of pooling. Every two convolutions, the upper input layer is reused in the output [37].

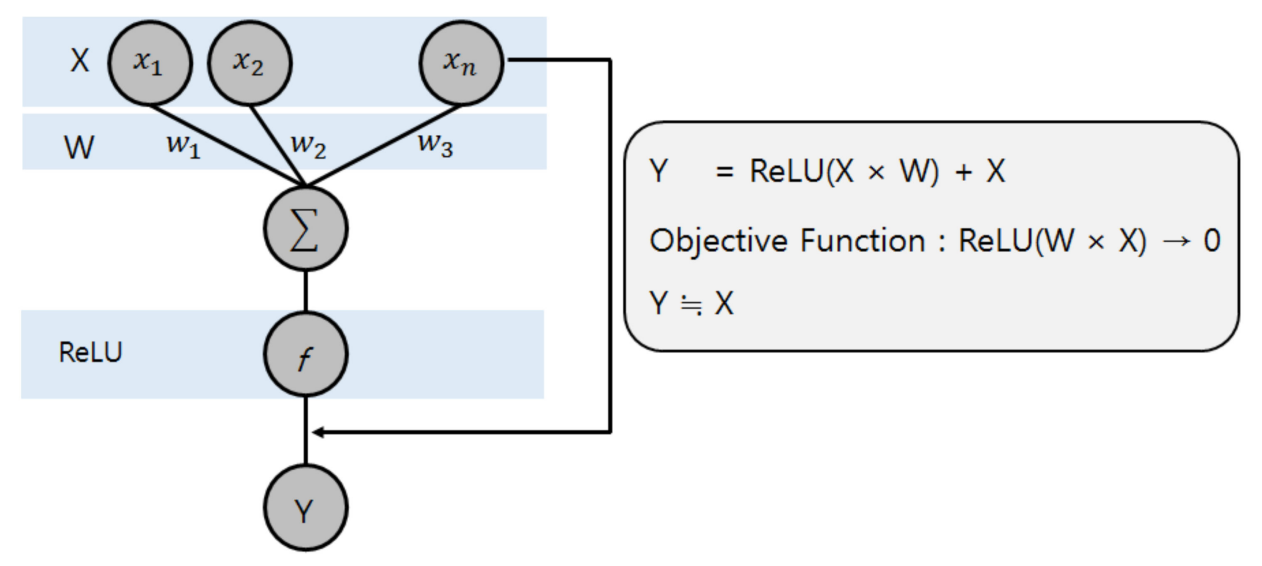

Figure 3. Principle of residual learning.

\section{Biometric Method using Scalogram of Electrocardiogram}

The biometric method is performed with 3 steps in this paper. First, the 1-D signals of electrocardiogram are preprocessed to eliminate noises. Secondly, the preprocessed signals become 2-D scalograms using CWT and Morse wavelet. By transforming the ECG signal from the time domain to the frequency domain using the CWT and Morse wavelet, it can be analyzed on multiresolution. Thirdly, deep models are trained with input of the scalograms.

\subsection{Preprocessing}

Noise occurs when measuring the ECG signal, which is caused by low-frequency noise due to muscle movement and high-frequency noise of 50 to $60 \mathrm{~Hz}$, depending on the power source used [41]. Low-frequency noise is removed by a convolution using an average filter of 500 filter size, and high-frequency noise is removed by convolution using an average filter of 10 filter size. Six-hundred frames are removed at the beginning and end of the signal, to remove the distorted parts due to filtering. After performing R-peak detection, 784 frames are obtained around the R-peak point. Only the I-lead of electrocardiogram was considered in this study [42].

\subsection{Continuous Wavelet Transform}

The wavelet transform is a more powerful method than the conventional cosine and Fourier transforms as a time-frequency transform [20]. Morlet introduced a wavelet as a family of translations and dilatations from a single function called the mother wavelet. This new signal processing has been 
improved more efficiently by Mallat, Meyer, Daubechies, and Grossman, and has become a popular technique in biosignal analysis. Instead of analyzing the Fourier transforms in a single scale (time or frequency), wavelet transforms are analyzed on a multiscale basis.

Wavelet is a windowing method with different resolutions for regions. Wavelet decomposition maps a signal into a time-scale plane using a scale instead of a frequency. This is the same as the time-frequency plane in the short-time Fourier transform (STFT) and each scale of the time-scale plane represents a certain frequency range of the time-frequency plane. A wavelet-a small waveform-is a localized wave for a limited duration. Comparing the wavelet with the Fourier transform, the Fourier analysis decomposes the signal into sinusoids of different frequencies, while the wavelet decomposes the signal into the shifted or scaled shapes from a mother wavelet.

The continuous wavelet transform (CWT) for the signal $f(t)$ is defined as integration of the $f(t)$ with the shifted or scaled shapes from a mother wavelet $\psi_{a, b}(t)$ :

$$
\begin{aligned}
\operatorname{CWT}(a, b) & =\frac{1}{\sqrt{a}} \int_{-\infty}^{+\infty} f(t) * \psi\left(\frac{t-b}{a}\right) d t \\
a & \in R^{+}-\{0\}, b \in R
\end{aligned}
$$

In other words, the CWT is the sum of the signal multiplied by shifted and scaled shapes from a mother wavelet $\psi$ :

$$
\operatorname{CWT}(\text { scale, position })=\int_{-\infty}^{+\infty} f(t) * \psi(\text { scale, position, } t) d t
$$

The original basic wavelet $\psi(t)$ is called the mother wavelet, and its variations $\psi_{a, b}(t)$ are called daughter wavelets. The daughter wavelets are the shifted or scaled shapes from a mother wavelet. The ' $a$ ' is scale factor for scaling the function $\psi(t)$, while the ' $b$ ' is a shift factor for translating the function $\psi(t)$. The result of the CWT is a matrix filled with wavelet coefficients located by scale and position. To determinate scale parameter and mother wavelet in CWT is very important for analyzing ECG [21].

\subsection{Morse Wavelet}

A wavelet means a small waveform satisfying two conditions. The first is that the amplitude of the signal rapidly decreases and converges to zero, and the second is that the function should oscillate. The wavelet filter has a feature that can decompose a signal into several frequencies and remove a specific frequency and amplitude. At this time, the role of the mother wavelet is important. There are several functions as mother wavelet. When the most similar wavelet with the signal to be decomposed is used, better noise cancellation without distortion can be performed [43].

A complex-valued wavelet in which the Fourier transform is only valid on the positive real axis is called an analytic wavelet, and a generalized Morse wavelet is its family. This is useful for analyzing signals with varying amplitude and frequency over time and localized discontinuities [44]. There have been many studies on Morse wavelet theory and its application to signal analysis [45-47], resulting in an efficient algorithm for calculating the Morse wavelet [48]. The Fourier transform of the generalized Morse wavelet is

$$
\Psi_{P, \gamma}(\omega)=U(\omega) a_{P, \gamma} \omega^{\frac{p^{2}}{\gamma}} e^{-\omega^{\gamma}}
$$

where, $U(\omega)$ is the function of unit step, $P^{2}$ is the time-bandwidth product, $a_{P, \gamma}$ is a constant for normalization, and $\gamma$ is a parameter for determining the symmetry of the Morse wavelet. In many applications of the Morse wavelet, $\beta$ is used as a decay or compactness parameter, rather than the time-bandwidth product, $P^{2}=\beta \gamma$ [45]. The equation for the Morse wavelet using $\beta$ and $\gamma$ as parameters is

$$
\Psi_{\beta, \gamma}(\omega)=U(\omega) a_{\beta, \gamma} \omega^{\beta} e^{-\omega^{\gamma}}
$$


Various analytic wavelets could be obtained by varying the time-bandwidth product and symmetry parameters of a Morse wavelet. Many useful analytic wavelets are a case of generalized Morse wavelets, such as Cauchy wavelets with $\gamma=1$ and Bessel wavelets with $\beta=8$ and $\gamma=0.25$.

The wavelet duration in time is proportional to the square root of $P$, which is the time-bandwidth product. The duration affects the number of oscillations of the center window at its peak frequency, $\left(\frac{P^{2}}{\gamma}\right)^{\frac{1}{\gamma}}$. The skewness of the Morse wavelet by demodulation is 0 when $\gamma$ is 3 as the minimum Heisenberg area. Therefore, the value is used in this study [49]. Figure 4 shows the Morse wavelet with $\gamma=3$ and $P^{2}=60$. Its signal length, sampling frequency, and voices per octave are 784,128 , and 12 , respectively. The number of voices per octave is used for discretizing the scales of CWT.

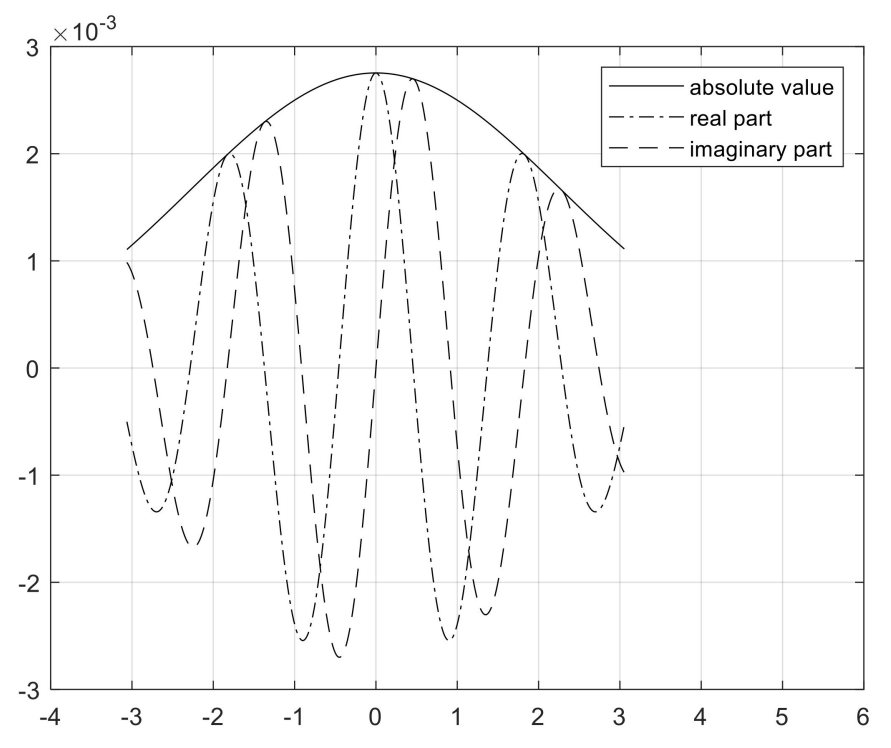

Figure 4. Morse wavelet with $\gamma=3$ and $P^{2}=60$.

\subsection{Biometrics using Scalogram of Electrocardiogram}

Since biometrics using electrocardiogram signals are sensitive to noise, studies have been conducted by converting signals into the frequency domain. Commonly used methods for the frequency analysis of signals are DCT, CWT, and DWT. A scalogram is the absolute value of the CWT coefficients of a signal. By transforming the signal from the time domain to the frequency domain using the wavelet the 1-D signal becomes a 2-D matrix, and it could be analyzed on multiresolution. The representation of the signal in the time domain is limited because it cannot represent the signal until infinite time. At this time, frequency-time analysis allows us to know how the signal is distributed with frequency and phase, so complex signals can be expressed concisely and analyzed easily. In other words, since the time-domain signals are noise and complex, different ECG signals may not be distinguishable due to lack of discriminatory features. However, by visually representing signals at various scales and various frequencies through CWT, hidden features can be seen in the frequency-time domain. The Morse wavelet with $r=3$ and $P^{2}=60$ is used as mother wavelet. However, this process makes morphologically complex signal analysis. This means that existing simple classifiers could be poor in performance. We investigate the possibility of using the image of frequency as input to CNN of deep learning, which exhibits optimal performance for the classification of morphological imagery. Figure 5 shows the scalogram of an electrocardiogram. Figure 6 shows biometrics using the scalogram of an electrocardiogram under varying the models of deep learning. 


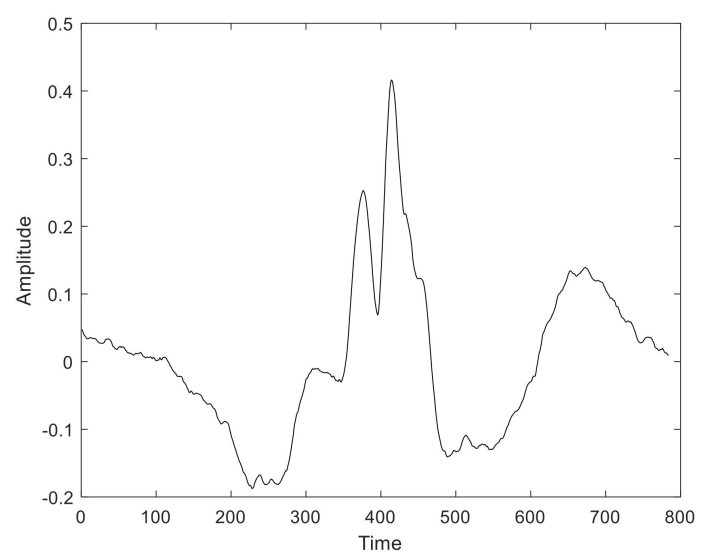

(a) original signal

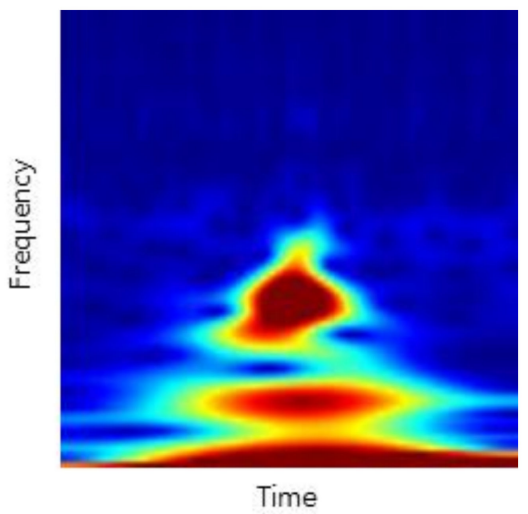

(b) scalogram by continuous wavelet transform

Figure 5. Scalogram of an electrocardiogram.

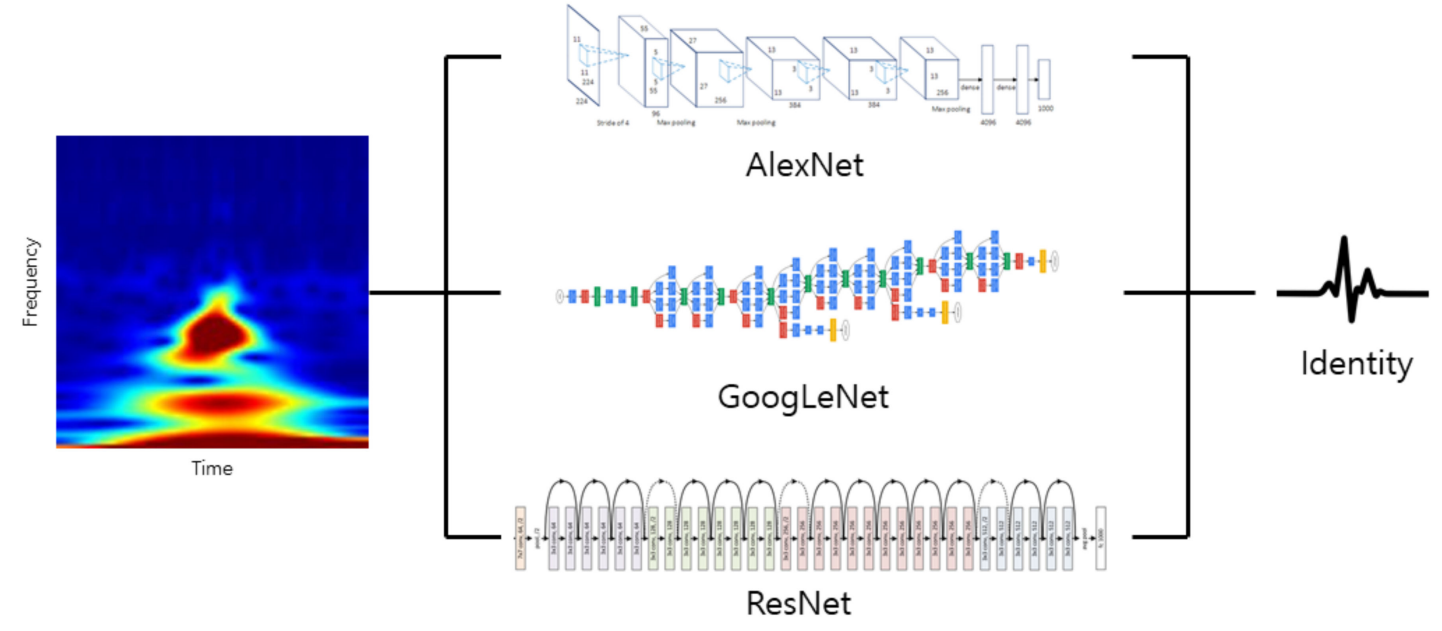

Scalogram of electrocardiogram

Applying to several models of deep learning

Individual identification

Figure 6. Biometrics using the scalogram of an electrocardiogram under varying the models of deep learning.

Here, accuracy is used to check the evaluation of the performance for biometrics. It is calculated by dividing the number of correct classification (CC) by the total number of classifications, the sum of CC, and the wrong classification (WC). The accuracy is defined as follows [50]

$$
\text { Accuracy }=\frac{C C}{C C+W C}
$$

In the verification stage, accuracy, sensitivity, specificity, false positive rate (FPR), and false negative rate (FNR) are considered as evaluation methods. True positive (TP) and true negative (TN) mean correctly classified as positive or negative. False positive (FP) and false negative (FN) mean incorrectly classified as positive or negative. Those methods are defined as follows [50]

$$
\begin{gathered}
\text { Accuracy }=\frac{\mathrm{TP}+\mathrm{TN}}{\mathrm{TP}+\mathrm{TN}+\mathrm{FP}+\mathrm{FN}} \\
\text { Sensitivity }=\frac{\mathrm{TP}}{\mathrm{TP}+\mathrm{FN}} \\
\text { Specificity }=\frac{\mathrm{TN}}{\mathrm{TN}+\mathrm{FP}}
\end{gathered}
$$




$$
\begin{aligned}
\mathrm{FPR} & =\frac{\mathrm{FP}}{\mathrm{TN}+\mathrm{FP}} \\
\mathrm{FNR} & =\frac{\mathrm{FN}}{\mathrm{TP}+\mathrm{FN}}
\end{aligned}
$$

\section{Experimental Result}

\subsection{Database}

Two databases were used for comparative analysis of deep models in biometrics using the scalogram of electrocardiogram. First, PTB-ECG is ECG data obtained from the National Metrology Institute of Physikalisch-Technische Bundesanstalt (PTB) in Germany, and contains 27,000 recordings. This PTB-ECG was obtained from 290 people sitting comfortably, including males and females. Some people had heart disease, and signals were measured from 15 leads at 1000 samples/s. The 15 leads are composed of 12 standard leads and three Frank leads. Each person has a different number of recordings, usually recorded two or three times, varying in length from 23 seconds to 2 minutes. After measuring the ECG once per person, the next measure was performed after 500 days on average [51,52]. Secondly, the CU-ECG is ECG data directly built for the biometrics at Chosun university (CU) in Korea. This database is obtained from 100 subjects (11 females and 89 males ranging from 23 to 34 years old). Sixty recordings per person were measured while subjects were sitting comfortably in a chair. The data were recorded for $10 \mathrm{~s}$ at a time, and only the lead-I signal was measured. The sampling rate of measure was $500 \mathrm{kHz}$. The measuring was performed using a directly developed device. The developed device consists of Keysight MSO9104 (Keysight technologies, Santa Rosa, CA, USA), Atmega8 (Microchip technology, Chandler, AZ, USA), and a wet-corrosion electrode. Figure 7 shows the developed device and environment for measuring ECG signals [15].

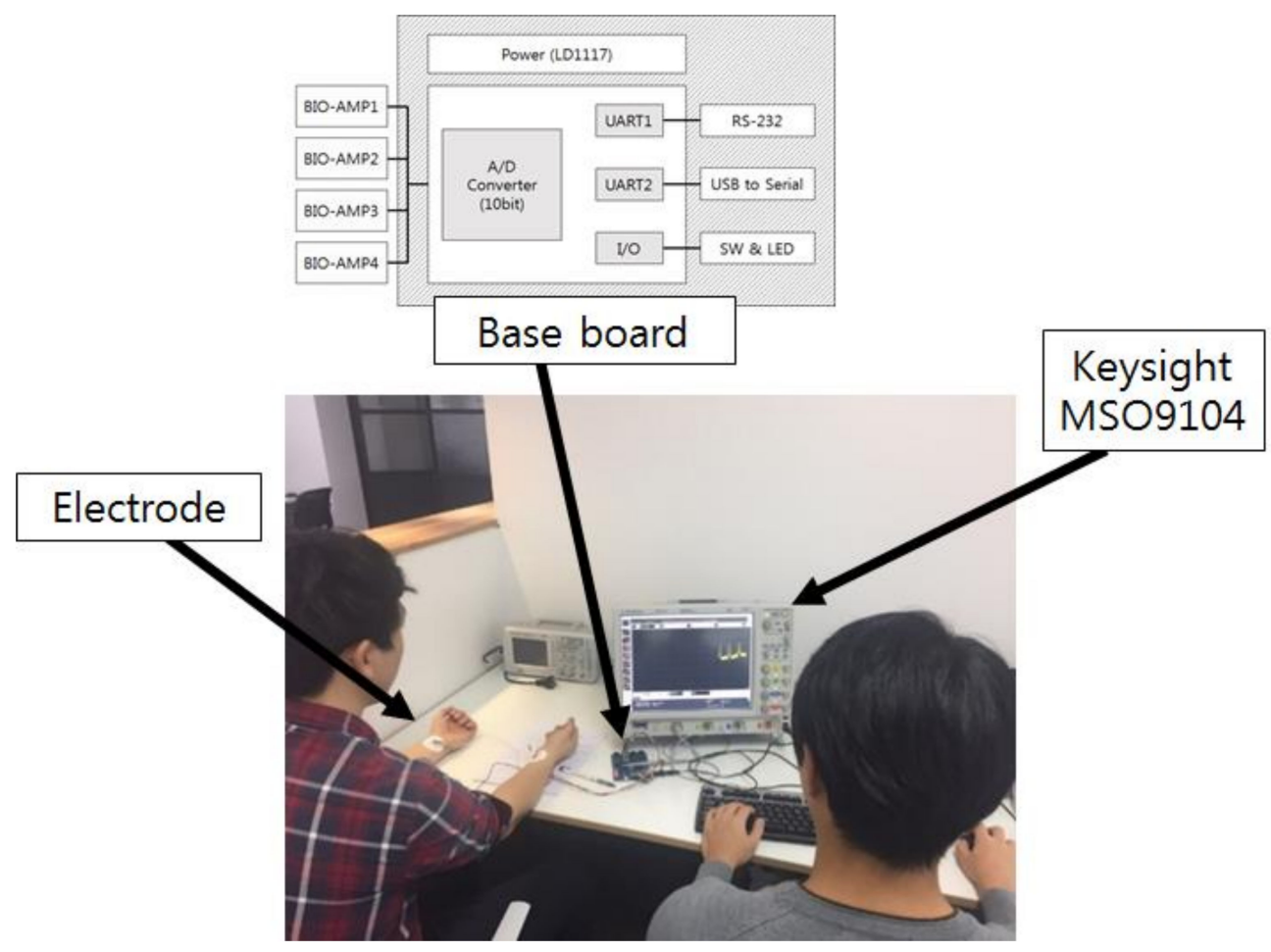

Figure 7. Developed device and environment for measuring ECG signals.

\subsection{Experimental Results}

The computer specification used in the experiment is Intel(R) Xeon(R) E5-1650 CPU (Central Processing Unit) v3 at 3.50 GHz, NVIDIA GeForce GTX Titan X, 32 GB RAM (Random 
Access Memory), and Window 7 64-bit OS (Operating System). In this study, the biometric of the electrocardiogram was performed by generating a scalogram from raw signal. Signals were preprocessed to eliminate noise, and then R-peak points were detected for all recordings. The number of R-peak points were identified for each class. To construct the same count of data, classes consisting of small R-peak points were excluded, and classes consisting of many R-peak points abandoned some detected R-peak points. Seven-hundred-and-forty-eight frames were obtained around the R-peak point, only near the I-lead of the electrocardiogram. The preprocessed ECG signals were then transformed to the scalograms using CWT and Morse wavelet. The wavelet-transformed scalograms were resized to $224 \times 224$ for normalization. Then classification was performed by using three models of deep learning. In the case of PTB-ECG, the 79 people consisting of small R-peak points were abandoned among 290 people. The number of people is the number of classes to be identified. The common maximum count of R-peak points among 211 people was 120 . Therefore, the database consisted of 120 data per class. The size of the constructed database was $784 \times 25,320$ ( 120 data/class $\times 211$ classes); the row presents the dimension of the data, while the column presents the number of data. The sizes of the data for training and test were $784 \times 12,660$ each, because the ratio of training was $50 \%$, and then the 1D signals were transformed to scalograms. Figure 8 shows the scalograms of PTB-ECG.

Simple CNN was tested as conventional method. It is composed of an input layer, three convolutional layers, two max-pooling layers, and one fully-connected layer. In first layer, an input layer takes $28 \times$ $28 \times 3$ image. In the second layer, a convolutional layer with 3,8 , and 1 as the filter size, number of filters, and number of padding size extracts features, respectively. In the third layer, a max-pooling layer with 2, 2 as filter size, stride is performed. In the fourth layer, a convolutional layer with 3,16, and 1 as the filter size, number of filters, and number of padding size extracts features, respectively. In the fifth layer, a max-pooling layer with 2, 2 as filter size, stride is performed. In the sixth layer, a convolutional layer with 3, 32, and 1 as the filter size, number of filters, and number of padding size extracts features, respectively. Finally, the fully-connected layer and softmax layer are performed.

The Stochastic Gradient Descent Method (SGDM), RMSProp, and Adaptive Moment Estimation (Adam) were used as learning methods. The basic learning method is a SGDM that moves in the opposite direction to the derivative direction from a group of random samples. Learning methods derived from SGDM are RMSProp and Adam. Their main strategy is to update parameters with a moderate learning rate at the beginning of learning and to reduce the learning rate as they approach the solution. Adam combines the advantages of momentum and RMSProp. In general, the loss function oscillates up and down, but it decreases as a whole during learning. The momentum prevents the current gradient from changing too much using the past gradient [53]. The initial learning rate was 0.0001 , and the recognition rate was confirmed by changing the size of minibatch. The multiplier for the learning rate was the multiplication of the learning rate for the weight and bias of the last fully connected layer. The epochs of learning were applied 5, 10, and 20 times. The range of transferred units, whether to import parameter values of the already learned deep learning model or not, was specified by transferred units; the $\mathrm{X}$ means learning without transfer. The indices of the last unit of AlexNet, GoogLeNet, and ResNet were 25, 144, and 347, respectively. Training data was divided into training and validation data again. Figure 9 shows data of training, validation, and test. The validation accuracy is a recognition rate for confirming the learning state during training. It helps determine whether overfitting occurs or not. If the difference of accuracy between training and validation is large, it means overfitting occurs. The test accuracy is a recognition rate when new data is input after the completion of learning. Recently, Lee proposed EECGNet for personal identification. For comparing performance with the state of the art, an additional experiment is performed using EECGNet with input of scalogram. We used patch size (K), the number of filters (L), block size (h), and overlap ratio of block (R) as parameters of EECGNet. For more detail is in Lee [54]. Tables 1-5 show the accuracies of simple CNN, AlexNet, GoogLeNet, ResNet, and EECGNet on PTB-ECG, respectively. In the case of PTB-ECG, the highest accuracy of simple CNN was $86.02 \%$ when the training method, minibatch size, transferred units, and epoch were (Adam, 200, X, and 13), respectively; the highest accuracy of AlexNet was $97.37 \%$ when the training method, minibatch size, 
transferred units, and epoch were (Adam, 100, X, and 20), respectively; the highest accuracy of GoogLeNet was $97.83 \%$ when the training method, minibatch size, transferred units, and epoch were Adam, 30, X, and 20, respectively; the highest accuracy of ResNet was $98.10 \%$ when the training method, minibatch size, transferred units, and epoch were RMSProp, 30, X, and 10, respectively. The ResNet was $0.73 \%-0.27 \%$ higher than AlexNet or GoogLeNet on PTB-ECG. Figure 10 shows the training processes in PTB-ECG. Figure 11 shows a comparison of the activations of the first and last convolutions in PTB-ECG. Figure 12 shows a comparison of the activations of the last ReLU by class in PTB-ECG. Figures 10-12 were recorded when the multiplier of learning rate, minibatch size, transferred units, and epoch were 10, 30, X, and 5, respectively, in training.

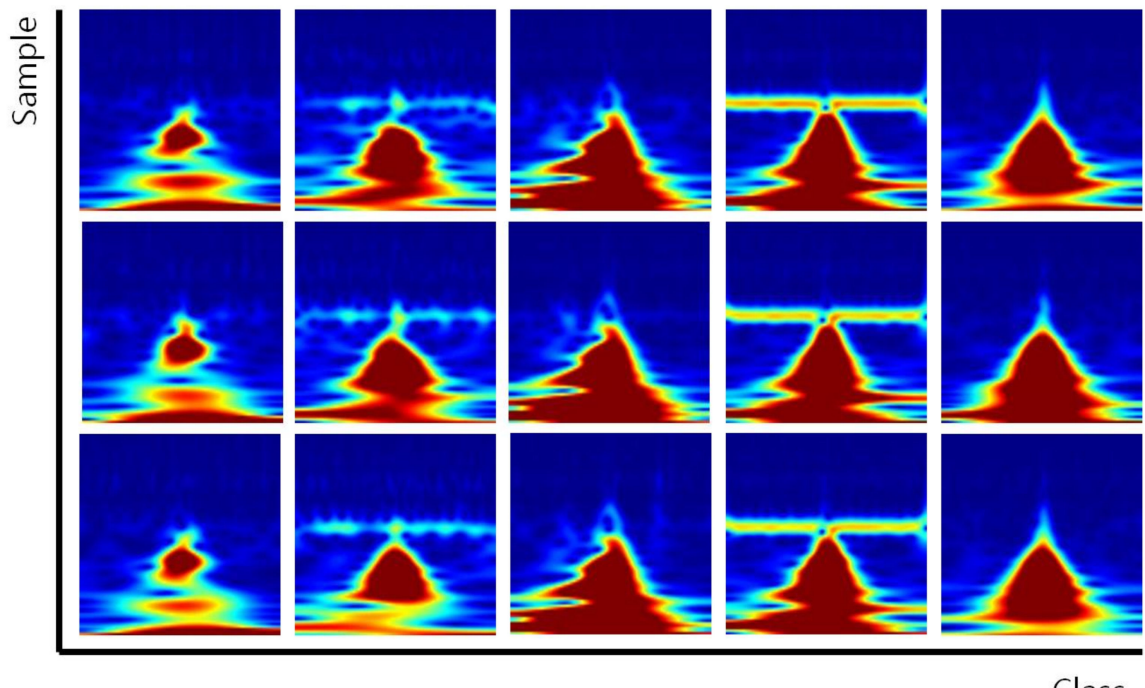

Figure 8. Scalograms of PTB-ECG.

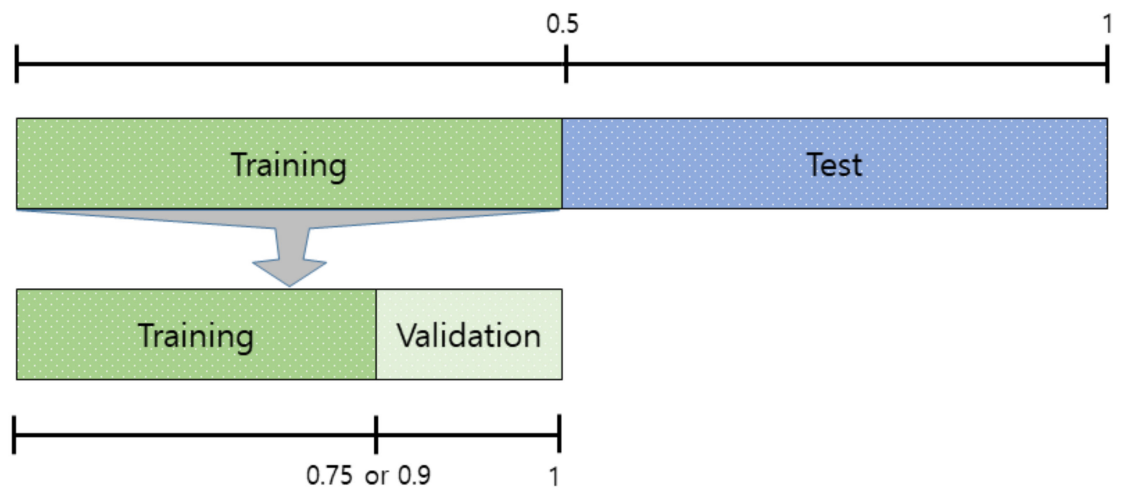

Figure 9. Data of training, validation, and test.

Table 1. Performance of simple CNN (convolutional neural network) on PTB-ECG.

\begin{tabular}{|c|c|c|c|c|c|c|c|}
\hline $\begin{array}{l}\text { Training } \\
\text { Method }\end{array}$ & $\begin{array}{c}\text { Ratio of } \\
\text { Training and } \\
\text { Validation }\end{array}$ & $\begin{array}{l}\text { Minibatch } \\
\text { Size }\end{array}$ & $\begin{array}{l}\text { Multiplier of } \\
\text { Learning Rate }\end{array}$ & Epoch & $\begin{array}{c}\text { Transferred } \\
\text { Units }\end{array}$ & $\begin{array}{l}\text { Validation } \\
\text { Accuracy }\end{array}$ & $\begin{array}{c}\text { Test } \\
\text { Accuracy }\end{array}$ \\
\hline SGDM & $90: 10$ & 30 & 10 & 5 & $x$ & 18.56 & 18.05 \\
\hline RMSProp & $90: 10$ & 30 & 10 & 2 & $X$ & 41.07 & 39.11 \\
\hline Adam & $90: 10$ & 20 & 10 & 2 & $X$ & 48.66 & 46.54 \\
\hline Adam & $90: 10$ & 30 & 10 & 2 & $X$ & 58.53 & 56.11 \\
\hline Adam & $90: 10$ & 40 & 10 & 3 & $X$ & 61.14 & 59.39 \\
\hline Adam & $90: 10$ & 50 & 10 & 3 & $x$ & 74.09 & 71.22 \\
\hline Adam & $90: 10$ & 64 & 10 & 6 & $X$ & 86.73 & 83.69 \\
\hline Adam & $90: 10$ & 100 & 10 & 8 & $X$ & 87.05 & 85.10 \\
\hline Adam & $90: 10$ & 200 & 10 & 10 & $X$ & 86.65 & 83.56 \\
\hline Adam & $90: 10$ & 200 & 10 & 13 & $x$ & 89.34 & 86.02 \\
\hline
\end{tabular}


Table 2. Performance of AlexNet on PTB-ECG.

\begin{tabular}{cccccccc}
\hline $\begin{array}{c}\text { Training } \\
\text { Method }\end{array}$ & $\begin{array}{c}\text { Ratio of } \\
\text { Training and } \\
\text { Validation }\end{array}$ & $\begin{array}{c}\text { Minibatch } \\
\text { Size }\end{array}$ & $\begin{array}{c}\text { Multiplier of } \\
\text { Learning Rate }\end{array}$ & Epoch & $\begin{array}{c}\text { Transferred } \\
\text { Units }\end{array}$ & $\begin{array}{c}\text { Validation } \\
\text { Accuracy }\end{array}$ & $\begin{array}{c}\text { Test } \\
\text { Accuracy }\end{array}$ \\
\hline SGDM & $90: 10$ & 30 & 10 & 5 & $1-22$ & 75.99 & 72.74 \\
SGDM & $90: 10$ & 30 & 10 & 20 & $1-22$ & 86.73 & 84.57 \\
RMSProp & $90: 10$ & 30 & 10 & 5 & $1-22$ & 64.30 & 63.17 \\
RMSProp & $90: 10$ & 30 & 10 & 20 & $1-22$ & 58.29 & 55.70 \\
Adam & $75: 25$ & 20 & 10 & 5 & $1-22$ & 63.38 & 61.97 \\
Adam & $75: 25$ & 30 & 10 & 5 & $1-22$ & 68.94 & 67.14 \\
Adam & $50: 50$ & 30 & 10 & 10 & $X$ & 97.54 & 95.77 \\
Adam & $75: 25$ & 30 & 10 & 20 & $1-22$ & 85.34 & 84.12 \\
Adam & $90: 10$ & 30 & 10 & 20 & $X$ & 98.89 & 97.37 \\
Adam & $75: 25$ & 40 & 10 & 20 & $1-22$ & 85.47 & 83.64 \\
Adam & $90: 10$ & 50 & 10 & 20 & $1-22$ & 86.10 & 83.73 \\
Adam & $90: 10$ & 100 & 10 & 20 & $1-22$ & 87.28 & 85.84 \\
\hline
\end{tabular}

Table 3. Performance of googleNet on PTB-ECG.

\begin{tabular}{|c|c|c|c|c|c|c|c|}
\hline $\begin{array}{l}\text { Training } \\
\text { Method }\end{array}$ & $\begin{array}{c}\text { Ratio of } \\
\text { Training and } \\
\text { Validation }\end{array}$ & $\begin{array}{l}\text { Minibatch } \\
\text { Size }\end{array}$ & $\begin{array}{l}\text { Multiplier of } \\
\text { Learning Rate }\end{array}$ & Epoch & $\begin{array}{c}\text { Transferred } \\
\text { Units }\end{array}$ & $\begin{array}{l}\text { Validation } \\
\text { Accuracy }\end{array}$ & $\begin{array}{c}\text { Test } \\
\text { Accuracy }\end{array}$ \\
\hline SGDM & $90: 10$ & 30 & 10 & 5 & $1-110$ & 89.81 & 86.94 \\
\hline SGDM & $90: 10$ & 30 & 10 & 20 & $1-110$ & 95.02 & 94.40 \\
\hline RMSProp & $90: 10$ & 30 & 10 & 5 & $1-110$ & 97.16 & 94.45 \\
\hline RMSProp & $90: 10$ & 30 & 10 & 20 & $1-110$ & 97.39 & 94.94 \\
\hline Adam & $90: 10$ & 30 & 10 & 5 & $X$ & 97.87 & 95.14 \\
\hline Adam & $90: 10$ & 30 & 10 & 5 & $1-110$ & 96.21 & 93.96 \\
\hline Adam & $90: 10$ & 30 & 10 & 10 & $1-110$ & 96.37 & 94.48 \\
\hline Adam & $50: 50$ & 30 & 10 & 10 & $x$ & 97.35 & 95.50 \\
\hline Adam & $90: 10$ & 30 & 10 & 20 & $1-110$ & 98.10 & 95.51 \\
\hline Adam & $90: 10$ & 30 & 10 & 20 & $X$ & 99.37 & 97.83 \\
\hline Adam & $90: 10$ & 40 & 10 & 5 & $1-110$ & 95.10 & 93.84 \\
\hline Adam & $90: 10$ & 50 & 10 & 5 & $1-110$ & 94.63 & 92.03 \\
\hline Adam & $90: 10$ & 100 & 10 & 5 & $1-110$ & 95.58 & 93.38 \\
\hline
\end{tabular}

Table 4. Performance of ResNet on PTB-ECG.

\begin{tabular}{|c|c|c|c|c|c|c|c|}
\hline $\begin{array}{l}\text { Training } \\
\text { Method }\end{array}$ & $\begin{array}{c}\text { Ratio of } \\
\text { Training and } \\
\text { Validation }\end{array}$ & $\begin{array}{c}\text { Minibatch } \\
\text { Size }\end{array}$ & $\begin{array}{l}\text { Multiplier of } \\
\text { Learning Rate }\end{array}$ & Epoch & $\begin{array}{c}\text { Transferred } \\
\text { Units }\end{array}$ & $\begin{array}{l}\text { Validation } \\
\text { Accuracy }\end{array}$ & $\begin{array}{c}\text { Test } \\
\text { Accuracy }\end{array}$ \\
\hline SGDM & $90: 10$ & 30 & 10 & 5 & $1-310$ & 93.68 & 91.97 \\
\hline SGDM & $90: 10$ & 30 & 10 & 10 & $1-310$ & 98.03 & 96.28 \\
\hline RMSProp & $90: 10$ & 30 & 10 & 5 & $1-310$ & 98.97 & 97.19 \\
\hline RMSProp & $90: 10$ & 30 & 10 & 10 & $1-310$ & 99.61 & 97.95 \\
\hline RMSProp & $90: 10$ & 30 & 10 & 10 & $x$ & 99.37 & 98.10 \\
\hline Adam & $90: 10$ & 30 & 10 & 5 & $1-310$ & 98.42 & 96.11 \\
\hline Adam & $90: 10$ & 30 & 10 & 10 & $1-310$ & 98.66 & 96.96 \\
\hline Adam & $50: 50$ & 30 & 10 & 5 & $x$ & 96.82 & 95.07 \\
\hline Adam & $50: 50$ & 30 & 10 & 10 & $x$ & 97.30 & 95.69 \\
\hline Adam & $90: 10$ & 30 & 10 & 10 & $X$ & 99.13 & 97.60 \\
\hline Adam & $90: 10$ & 40 & 10 & 5 & $1-310$ & 98.34 & 96.48 \\
\hline Adam & $90: 10$ & 50 & 10 & 5 & $1-310$ & 98.10 & 96.14 \\
\hline Adam & $90: 10$ & 100 & 10 & 5 & $1-310$ & 98.34 & 96.79 \\
\hline
\end{tabular}

Table 5. Performance of EECGNet on PTB-ECG.

\begin{tabular}{cccccc}
\hline K & L & h & R & Training Accuracy & Test Accuracy \\
\hline 4 & 4 & 4 & 0.5 & 99.78 & 98.89 \\
8 & 4 & 8 & 0.5 & 99.72 & 98.04 \\
12 & 8 & 12 & 0.5 & 99.72 & 98.15 \\
\hline
\end{tabular}




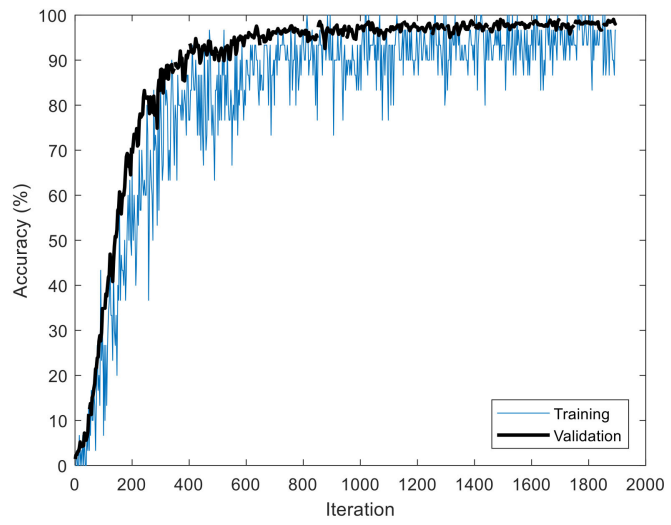

(a) Accuracy on AlexNet

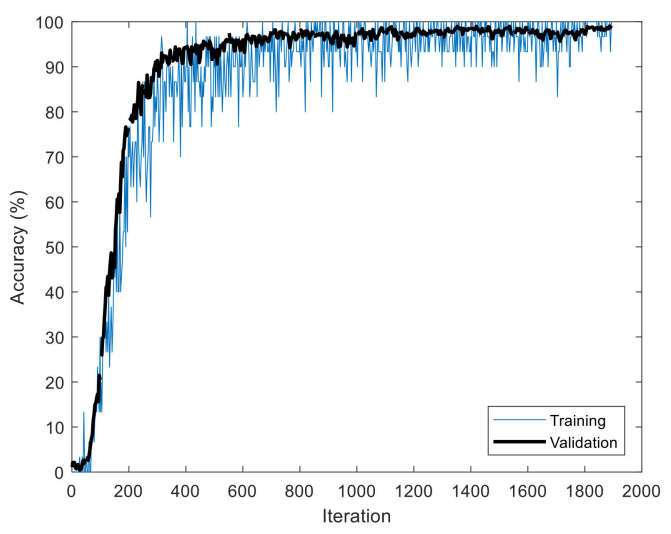

(c) Accuracy on GoogLeNet

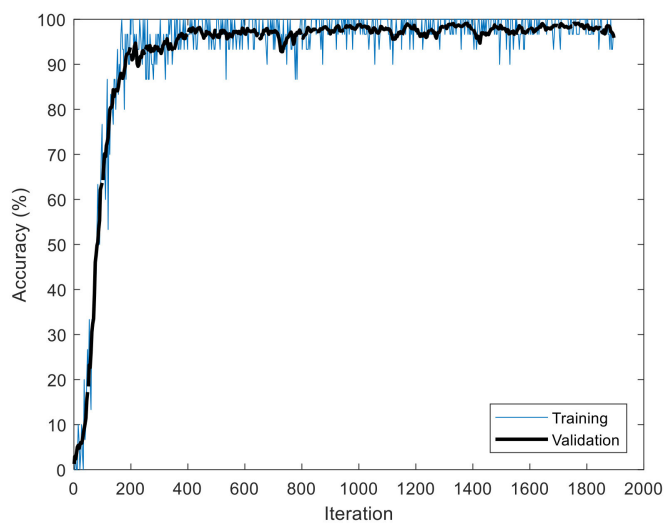

(e) Accuracy on ResNet

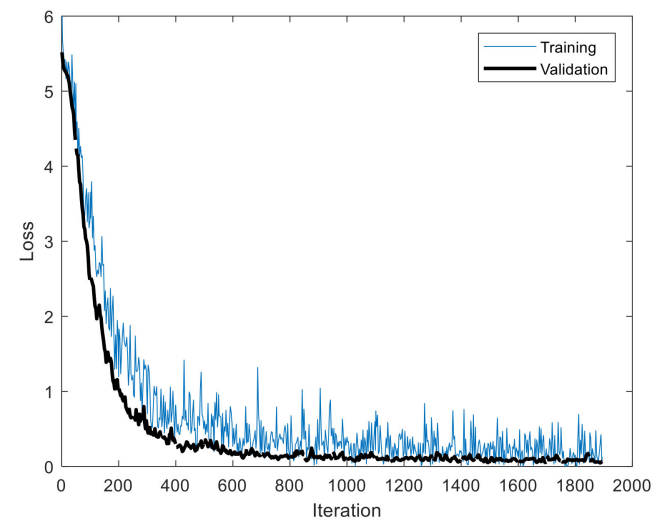

(b) Loss on AlexNet

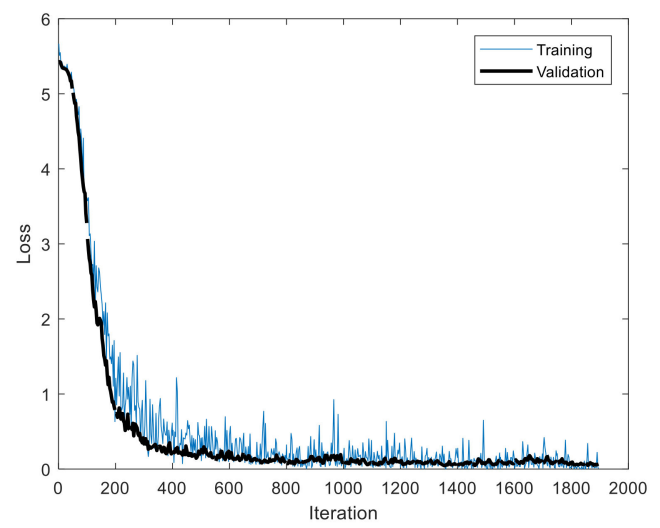

(d) Loss on GoogLeNet

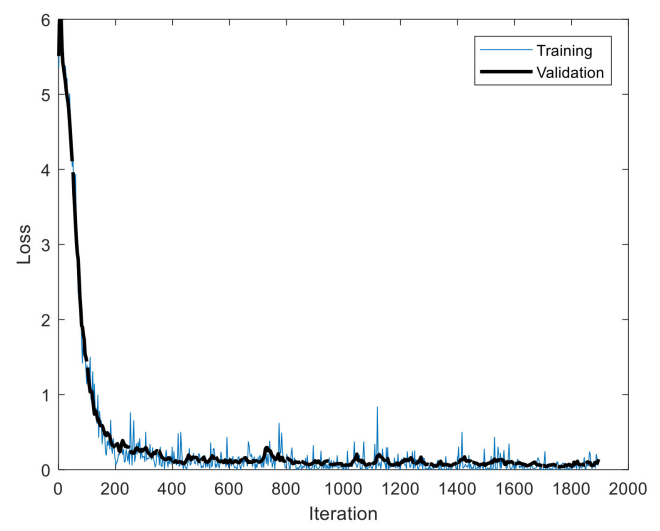

(f) Loss on ResNet

Figure 10. Training processes in PTB-ECG. 


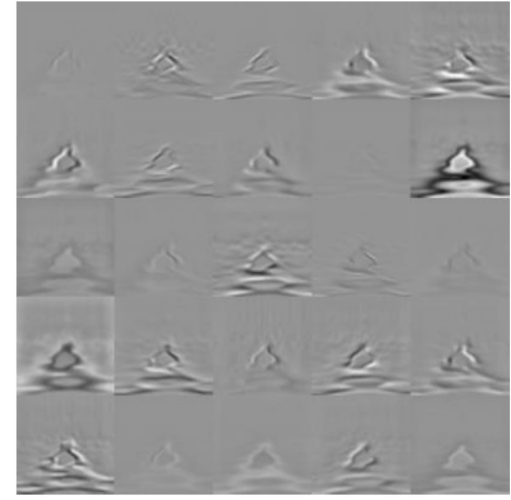

(a) Activations of first convolution on AlexNet

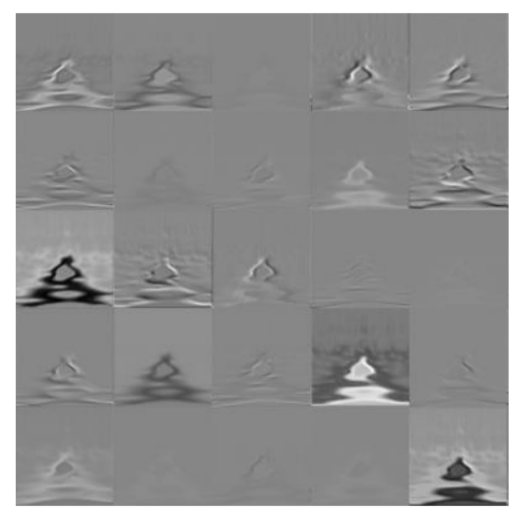

(c) Activations of first convolution on GoogLeNet

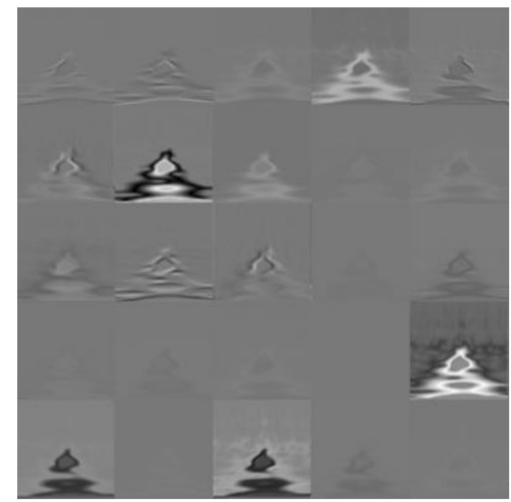

(e) Activations of first convolution on ResNet

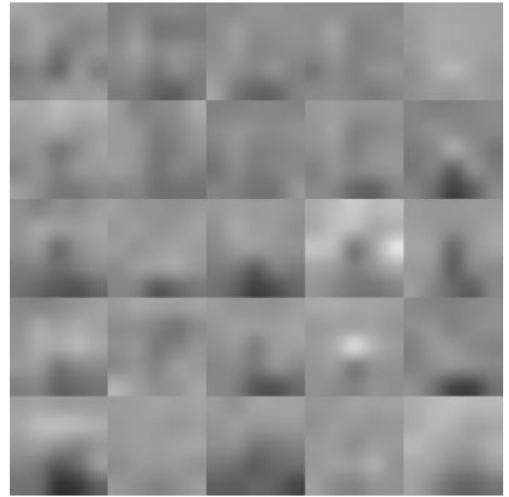

(b) Activations of last convolution on AlexNet

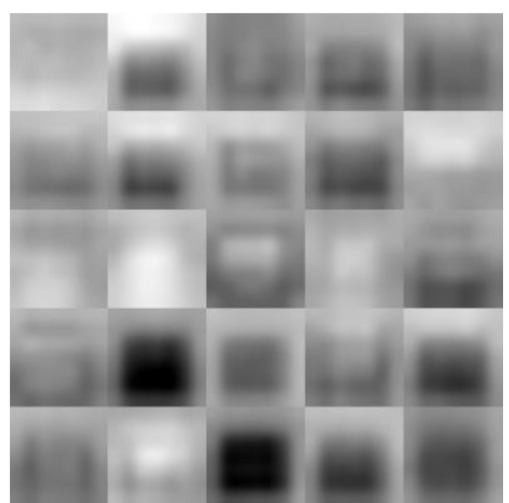

(d) Activations of last convolution on GoogLeNet

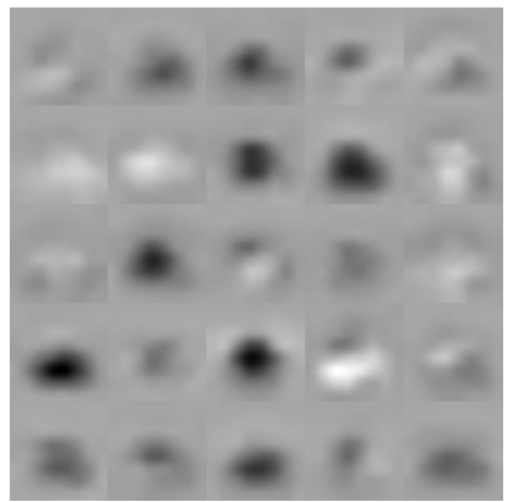

(f) Activations of last convolution on ResNet

Figure 11. Comparison of the activations of the first and last convolutions in PTB-ECG. 


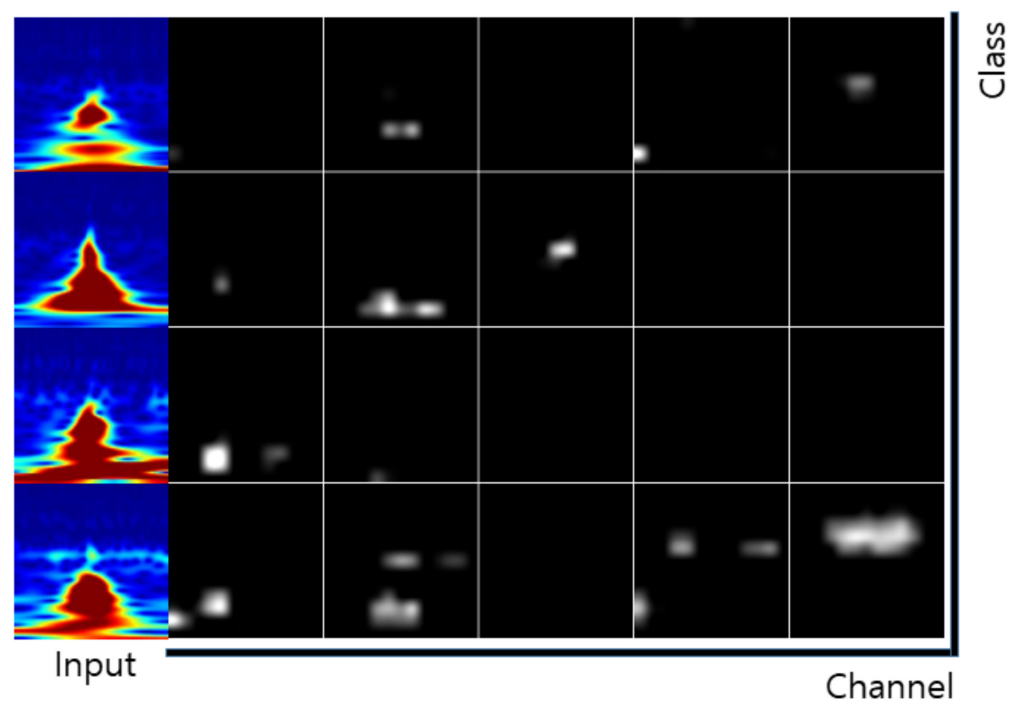

(a) Activations of last ReLU by class on AlexNet

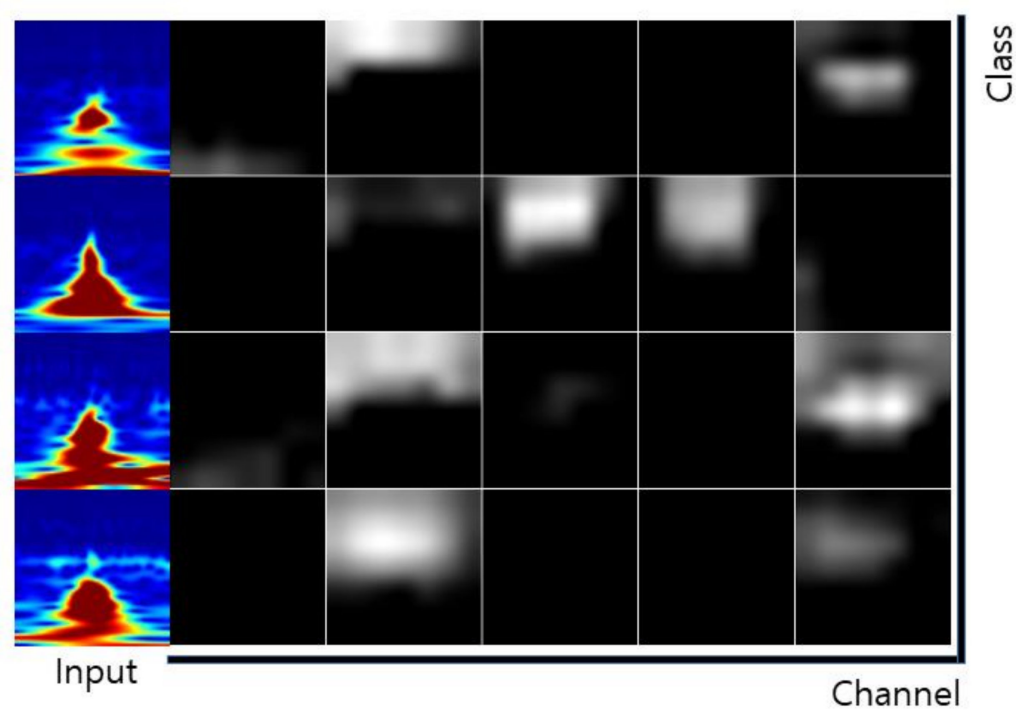

(b) Activations of last ReLU by class on GoogLeNet

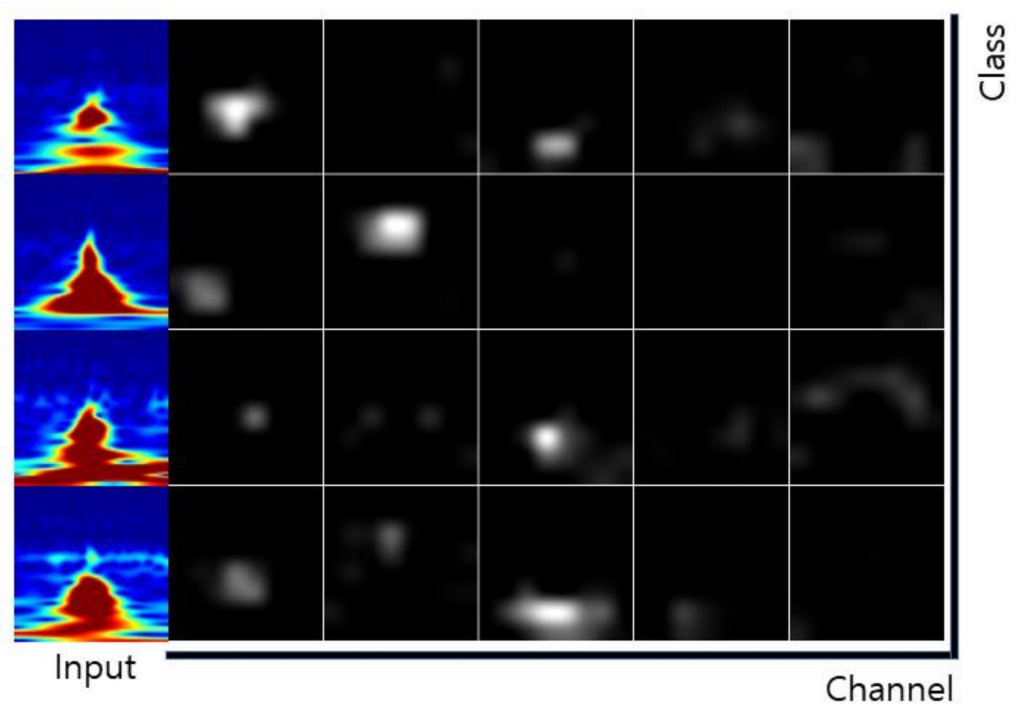

(c) Activations of last ReLU by class on ResNet

Figure 12. Comparison of the activations of the last ReLU (Rectified Linear Unit) by class in PTB-ECG. 
In the case of CU-ECG, the database was resampled from 500 to $1 \mathrm{kHz}$, because the data took much memory and processing load, and one person consisting of a small number of R-peak points was abandoned among the 100 people. The number of people is the number of classes to be identified. The common maximum count of R-peak points among 99 classes was 300 . Therefore, the database consisted of 300 data per class. The size of the constructed database was $784 \times 29,700$ (300 data/class $\times 99$ classes); the row presents the dimension of the data, while the column presents the number of data. The sizes of the data for training and test were $784 \times 14,850$ each, because the ratio of training was $50 \%$, and then the $1 \mathrm{D}$ signals were transformed to scalograms. Figure 13 shows the scalograms of CU-ECG. The initial learning rate was 0.0001 , and the recognition rate was confirmed by changing the size of minibatch. The epochs of learning were applied 5, 10, and 20 times. Tables 6-10 show the accuracies of simple CNN, AlexNet, GoogLeNet, ResNet, and EECGNet on CU-ECG, respectively. In the case of CU-ECG, the highest accuracy of simple $\mathrm{CNN}$ was $60.83 \%$ when the training method, minibatch size, transferred units, and epoch were Adam, 200, X, and 8, respectively; the highest accuracy of AlexNet was 92.26\% when the training method, minibatch size, transferred units, and epoch were Adam, 30, X, and 20, respectively; the highest accuracy of googleNet was $93.08 \%$ when the training method, minibatch size, transferred units, and epoch were Adam, 30, X, and 20, respectively; the highest accuracy of ResNet was 93.20\% when the training method, minibatch size, transferred units, and epoch were RMSProp, 30, X, and 10, respectively. The ResNet was $0.94 \%-0.12 \%$ higher than AlexNet or googleNet on CU-ECG. Figure 14 shows the training processes in CU-ECG. Figure 15 shows a comparison of the activations of the first and last convolutions in CU-ECG. Figure 16 shows a comparison of the activations of the last ReLU by class in CU-ECG. Figures 14-16 were recorded when the multiplier of learning rate, minibatch size, transferred units, and epoch were $10,30, \mathrm{X}$, and 5, respectively, in training.

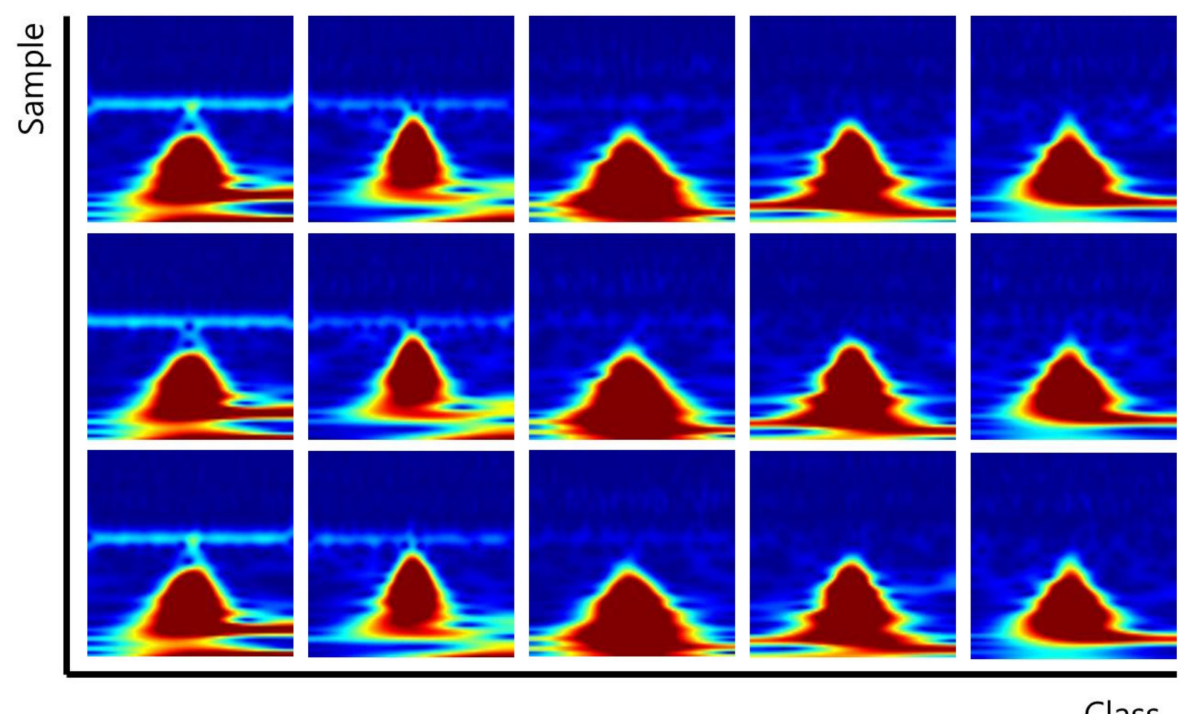

Figure 13. Scalograms of CU-ECG.

Table 6. Performance of simple CNN on CU-ECG.

\begin{tabular}{|c|c|c|c|c|c|c|c|}
\hline $\begin{array}{l}\text { Training } \\
\text { Method }\end{array}$ & $\begin{array}{c}\text { Ratio of } \\
\text { Training and } \\
\text { Validation }\end{array}$ & $\begin{array}{l}\text { Minibatch } \\
\text { Size }\end{array}$ & $\begin{array}{l}\text { Multiplier of } \\
\text { Learning Rate }\end{array}$ & Epoch & $\begin{array}{l}\text { Transferred } \\
\text { Units }\end{array}$ & $\begin{array}{l}\text { Validation } \\
\text { Accuracy }\end{array}$ & $\begin{array}{c}\text { Test } \\
\text { Accuracy }\end{array}$ \\
\hline SGDM & $90: 10$ & 30 & 10 & 3 & $X$ & 19.73 & 18.90 \\
\hline RMSProp & $90: 10$ & 30 & 10 & 1 & $X$ & 27.00 & 24.55 \\
\hline Adam & $90: 10$ & 20 & 10 & 1 & $X$ & 20.27 & 19.75 \\
\hline Adam & $90: 10$ & 30 & 10 & 1 & $X$ & 26.60 & 23.86 \\
\hline Adam & $90: 10$ & 40 & 10 & 2 & $X$ & 45.52 & 41.91 \\
\hline Adam & $90: 10$ & 50 & 10 & 2 & $X$ & 47.34 & 44.30 \\
\hline Adam & $90: 10$ & 64 & 10 & 2 & $X$ & 39.33 & 37.24 \\
\hline
\end{tabular}


Table 6. Cont.

\begin{tabular}{ccccccc}
\hline $\begin{array}{c}\text { Training } \\
\text { Method }\end{array}$ & $\begin{array}{c}\text { Ratio of } \\
\text { Training and } \\
\text { Validation }\end{array}$ & $\begin{array}{c}\text { Minibatch } \\
\text { Size }\end{array}$ & $\begin{array}{c}\text { Multiplier of } \\
\text { Learning Rate }\end{array}$ & Epoch & $\begin{array}{c}\text { Transferred } \\
\text { Units }\end{array}$ & $\begin{array}{c}\text { Validation } \\
\text { Accuracy }\end{array}$ \\
Accuracy \\
Adam & $90: 10$ & 100 & 10 & 4 & $X$ & 49.43 \\
Adam & $90: 10$ & 200 & 10 & 6 & $X$ & 56.16 \\
Adam & $90: 10$ & 200 & 10 & 8 & $X$ & 67.34 \\
\hline
\end{tabular}

Table 7. Performance of AlexNet on CU-ECG.

\begin{tabular}{|c|c|c|c|c|c|c|c|}
\hline $\begin{array}{l}\text { Training } \\
\text { Method }\end{array}$ & $\begin{array}{c}\text { Ratio of } \\
\text { Training and } \\
\text { Validation }\end{array}$ & $\begin{array}{l}\text { Minibatch } \\
\text { Size }\end{array}$ & $\begin{array}{l}\text { Multiplier of } \\
\text { Learning Rate }\end{array}$ & Epoch & $\begin{array}{c}\text { Transferred } \\
\text { Units }\end{array}$ & $\begin{array}{l}\text { Validation } \\
\text { Accuracy }\end{array}$ & $\begin{array}{c}\text { Test } \\
\text { Accuracy }\end{array}$ \\
\hline SGDM & $90: 10$ & 30 & 10 & 5 & $1-22$ & 47.21 & 44.05 \\
\hline SGDM & $90: 10$ & 30 & 10 & 20 & $1-22$ & 52.66 & 51.36 \\
\hline RMSProp & $90: 10$ & 30 & 10 & 5 & $1-22$ & 33.47 & 30.82 \\
\hline RMSProp & $90: 10$ & 30 & 10 & 20 & $1-22$ & 31.78 & 28.26 \\
\hline Adam & $90: 10$ & 30 & 10 & 5 & $1-22$ & 47.57 & 43.95 \\
\hline Adam & $50: 50$ & 30 & 10 & 10 & $X$ & 86.84 & 81.87 \\
\hline Adam & $90: 10$ & 30 & 10 & 10 & $1-22$ & 54.01 & 49.58 \\
\hline Adam & $90: 10$ & 30 & 10 & 20 & $1-22$ & 56.30 & 53.04 \\
\hline Adam & $90: 10$ & 30 & 10 & 20 & $X$ & 94.28 & 92.26 \\
\hline Adam & $90: 10$ & 40 & 10 & 20 & $1-22$ & 58.05 & 55.21 \\
\hline Adam & $90: 10$ & 50 & 10 & 20 & $1-22$ & 56.23 & 53.58 \\
\hline Adam & $90: 10$ & 100 & 10 & 20 & $1-22$ & 56.50 & 53.64 \\
\hline
\end{tabular}

Table 8. Performance of googleNet on CU-ECG.

\begin{tabular}{|c|c|c|c|c|c|c|c|}
\hline $\begin{array}{l}\text { Training } \\
\text { Method }\end{array}$ & $\begin{array}{c}\text { Ratio of } \\
\text { Training and } \\
\text { Validation }\end{array}$ & $\begin{array}{l}\text { Minibatch } \\
\text { Size }\end{array}$ & $\begin{array}{l}\text { Multiplier of } \\
\text { Learning Rate }\end{array}$ & Epoch & $\begin{array}{c}\text { Transferred } \\
\text { Units }\end{array}$ & $\begin{array}{l}\text { Validation } \\
\text { Accuracy }\end{array}$ & $\begin{array}{c}\text { Test } \\
\text { Accuracy }\end{array}$ \\
\hline SGDM & $90: 10$ & 30 & 10 & 5 & $1-110$ & 64.11 & 62.16 \\
\hline SGDM & $90: 10$ & 30 & 10 & 20 & $1-110$ & 81.75 & 78.45 \\
\hline RMSProp & $90: 10$ & 30 & 10 & 5 & $1-110$ & 85.05 & 70.79 \\
\hline RMSProp & $90: 10$ & 30 & 10 & 20 & $1-110$ & 89.70 & 84.94 \\
\hline Adam & $90: 10$ & 30 & 10 & 5 & $1-110$ & 82.76 & 79.55 \\
\hline Adam & $90: 10$ & 30 & 10 & 10 & $1-110$ & 89.23 & 85.12 \\
\hline Adam & $50: 50$ & 30 & 10 & 10 & $X$ & 88.46 & 83.75 \\
\hline Adam & $90: 10$ & 30 & 10 & 20 & $1-110$ & 89.83 & 86.71 \\
\hline Adam & $90: 10$ & 30 & 10 & 20 & $x$ & 95.35 & 93.08 \\
\hline Adam & $90: 10$ & 30 & 20 & 10 & $1-110$ & 89.23 & 84.68 \\
\hline Adam & $90: 10$ & 40 & 10 & 5 & $1-110$ & 84.65 & 81.23 \\
\hline Adam & $90: 10$ & 50 & 10 & 5 & $1-110$ & 82.69 & 79.58 \\
\hline Adam & $90: 10$ & 100 & 10 & 5 & $1-110$ & 81.41 & 76.57 \\
\hline
\end{tabular}

Table 9. Performance of ResNet on CU-ECG.

\begin{tabular}{|c|c|c|c|c|c|c|c|}
\hline $\begin{array}{l}\text { Training } \\
\text { Method }\end{array}$ & $\begin{array}{c}\text { Ratio of } \\
\text { Training and } \\
\text { Validation }\end{array}$ & $\begin{array}{l}\text { Minibatch } \\
\text { Size }\end{array}$ & $\begin{array}{l}\text { Multiplier of } \\
\text { Learning Rate }\end{array}$ & Epoch & $\begin{array}{l}\text { Transferred } \\
\text { Units }\end{array}$ & $\begin{array}{l}\text { Validation } \\
\text { Accuracy }\end{array}$ & $\begin{array}{c}\text { Test } \\
\text { Accuracy }\end{array}$ \\
\hline SGDM & $90: 10$ & 30 & 10 & 5 & $1-310$ & 85.86 & 82.25 \\
\hline SGDM & $90: 10$ & 30 & 10 & 10 & $1-310$ & 91.38 & 88.19 \\
\hline RMSProp & $90: 10$ & 30 & 10 & 5 & $1-310$ & 93.20 & 90.79 \\
\hline RMSProp & $90: 10$ & 30 & 10 & 10 & $1-310$ & 94.34 & 91.93 \\
\hline RMSProp & $90: 10$ & 30 & 10 & 10 & $x$ & 94.75 & 93.20 \\
\hline Adam & $90: 10$ & 30 & 10 & 5 & $1-310$ & 93.87 & 90.88 \\
\hline Adam & $50: 50$ & 30 & 10 & 5 & $X$ & 91.00 & 86.58 \\
\hline Adam & $90: 10$ & 30 & 10 & 10 & $1-310$ & 94.95 & 92.21 \\
\hline Adam & $90: 10$ & 30 & 10 & 20 & $1-310$ & 94.28 & 91.47 \\
\hline Adam & $90: 10$ & 30 & 20 & 10 & $1-310$ & 93.06 & 90.67 \\
\hline Adam & $90: 10$ & 30 & 30 & 10 & $1-310$ & 93.33 & 89.93 \\
\hline
\end{tabular}


Table 9. Cont.

\begin{tabular}{|c|c|c|c|c|c|c|c|}
\hline $\begin{array}{l}\text { Training } \\
\text { Method }\end{array}$ & $\begin{array}{c}\text { Ratio of } \\
\text { Training and } \\
\text { Validation }\end{array}$ & $\begin{array}{l}\text { Minibatch } \\
\text { Size }\end{array}$ & $\begin{array}{l}\text { Multiplier of } \\
\text { Learning Rate }\end{array}$ & Epoch & $\begin{array}{l}\text { Transferred } \\
\text { Units }\end{array}$ & $\begin{array}{l}\text { Validation } \\
\text { Accuracy }\end{array}$ & $\begin{array}{c}\text { Test } \\
\text { Accuracy }\end{array}$ \\
\hline Adam & $90: 10$ & 30 & 10 & 10 & $X$ & 94.21 & 91.74 \\
\hline Adam & $90: 10$ & 40 & 10 & 5 & $1-310$ & 91.25 & 89.45 \\
\hline Adam & $90: 10$ & 50 & 10 & 5 & $1-310$ & 89.90 & 87.74 \\
\hline Adam & $90: 10$ & 100 & 10 & 5 & $1-310$ & 93.20 & 90.88 \\
\hline
\end{tabular}

Table 10. Performance of EECGNet on CU-ECG.

\begin{tabular}{cccccc}
\hline K & L & h & R & Training Accuracy & Test Accuracy \\
\hline 4 & 4 & 4 & 0.5 & 95.92 & 92.29 \\
8 & 4 & 8 & 0.5 & 95.72 & 86.96 \\
12 & 8 & 12 & 0.5 & 95.88 & 89.50 \\
\hline
\end{tabular}

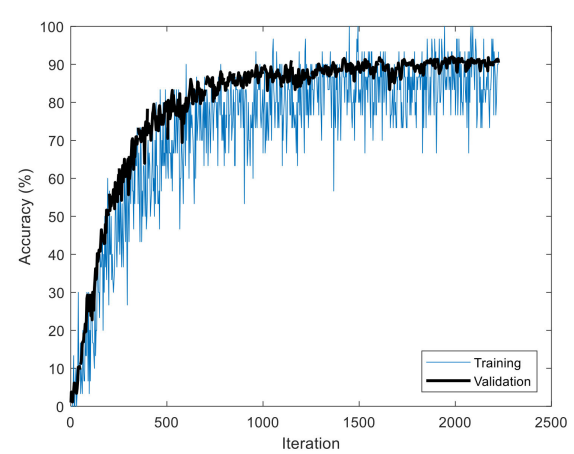

(a) Accuracy on AlexNet

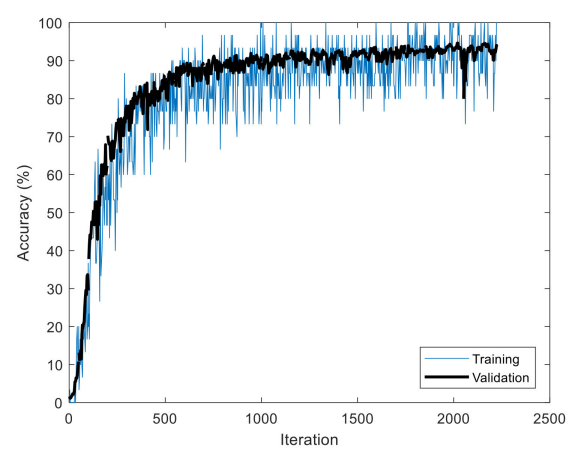

(c) Accuracy on GoogLeNet

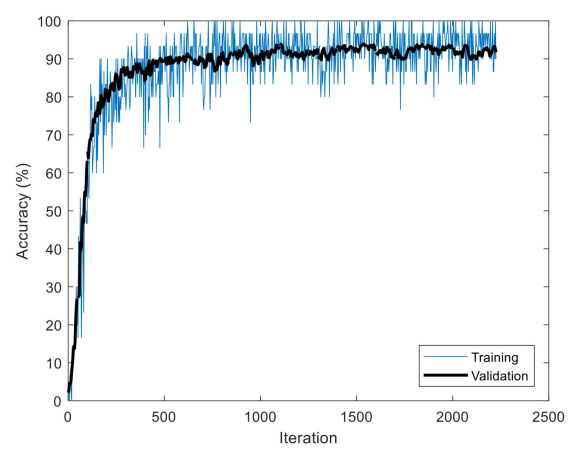

(e) Accuracy on ResNet

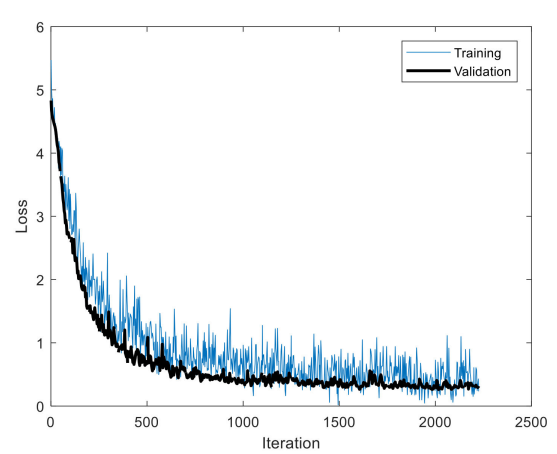

(b) Loss on AlexNet

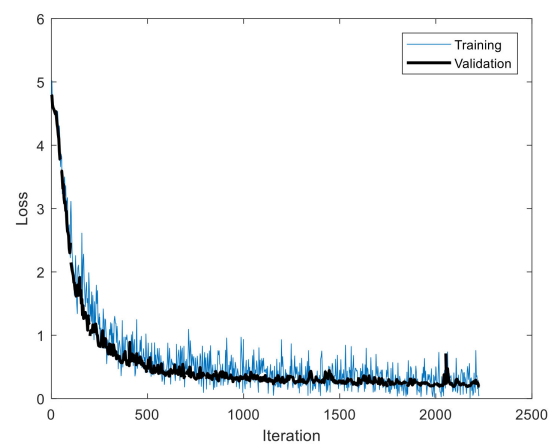

(d) Loss on GoogLeNet

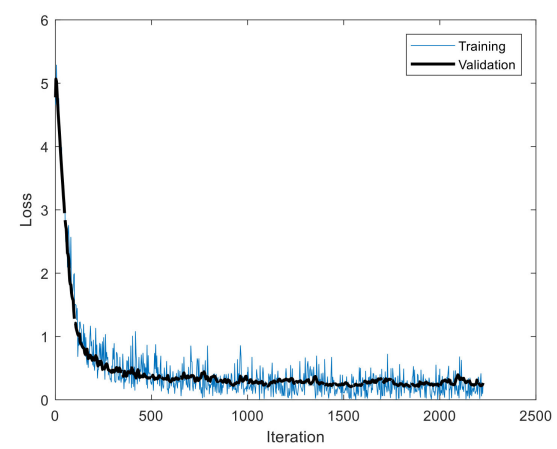

(f) Loss on ResNet

Figure 14. Training processes in CU-ECG. 


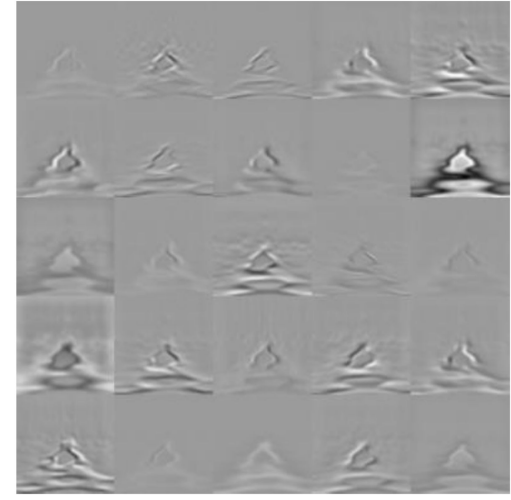

(a) Activations of first convolution on AlexNet

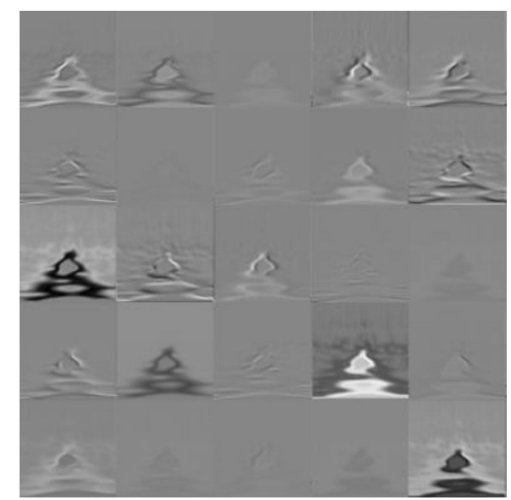

(c) Activations of first convolution on googleNet

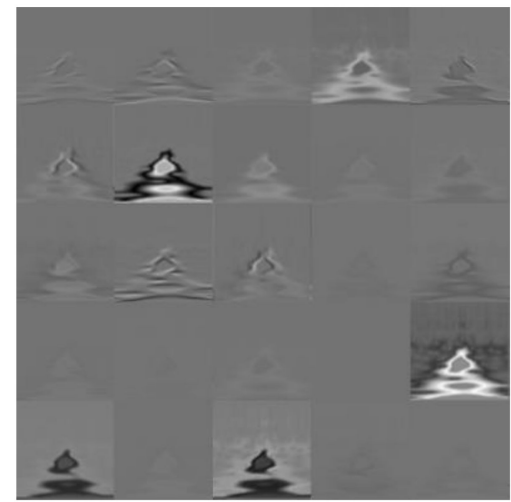

(e) Activations of first convolution on ResNet

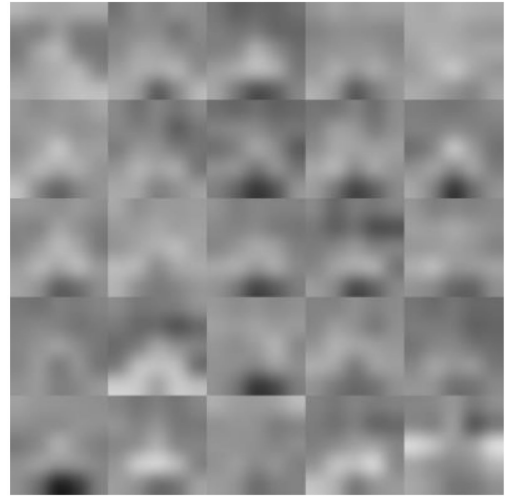

(b) Activations of last convolution on AlexNet

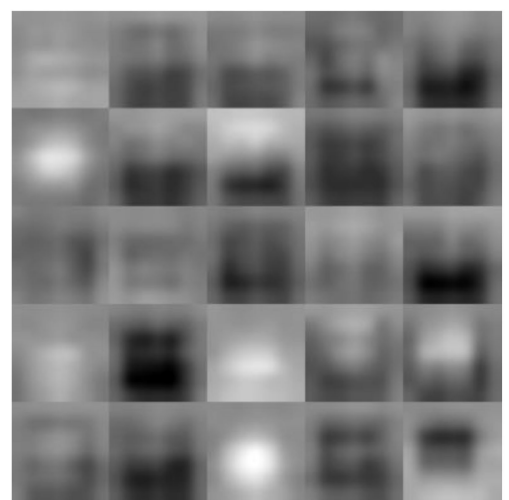

(d) Activations of last convolution on googleNet

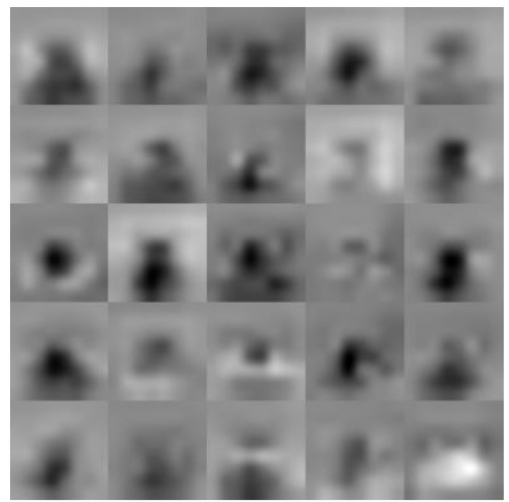

(f) Activations of last convolution on ResNet

Figure 15. Comparison of the activations of the first and last convolutions in CU-ECG. 


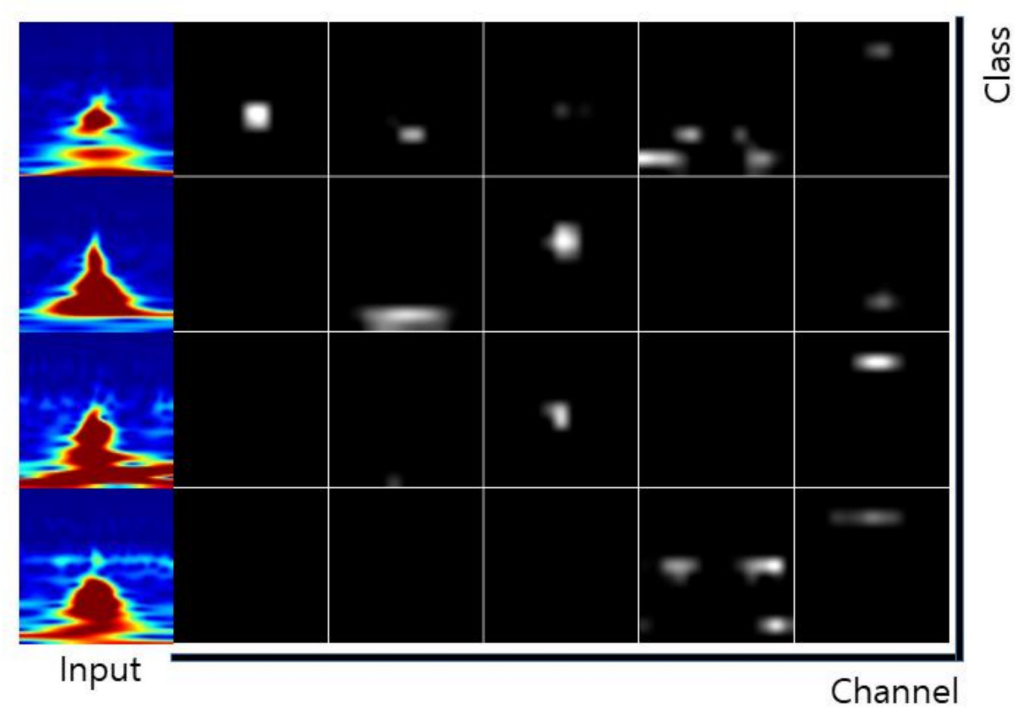

(a) Activations of last ReLU by class on AlexNet

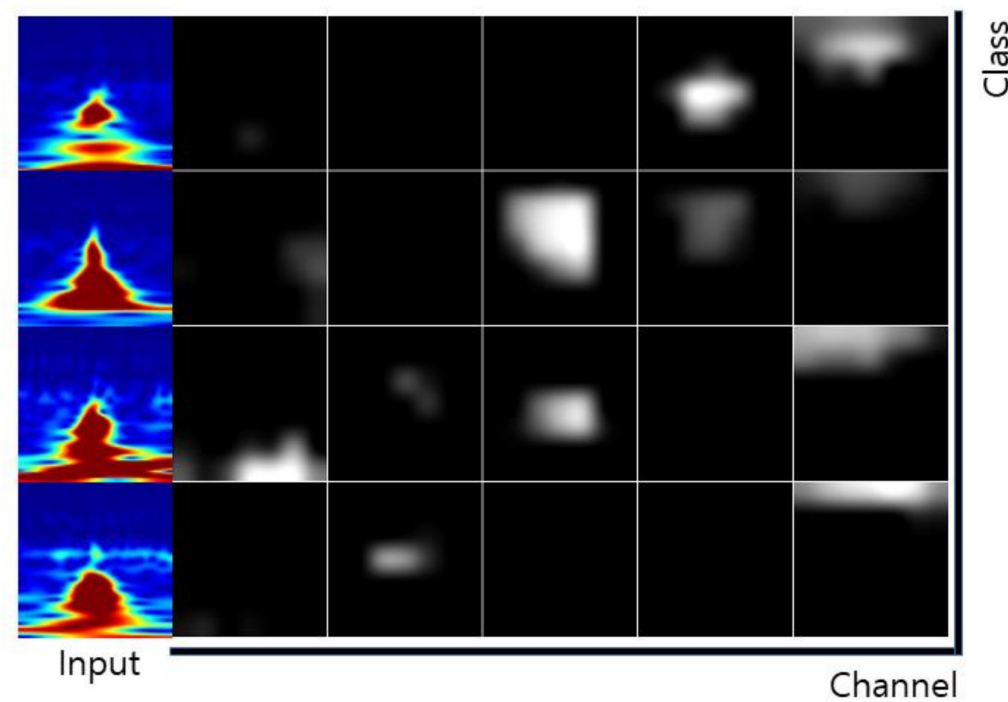

(b) Activations of last ReLU by class on googleNet

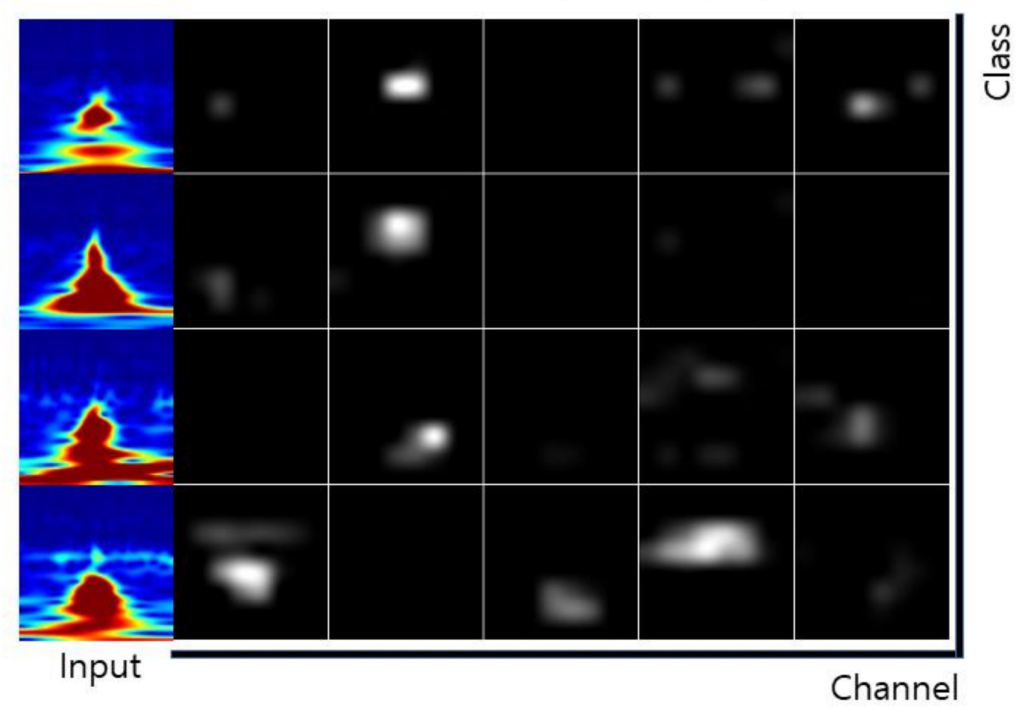

(c) Activations of last ReLU by class on ResNet

Figure 16. Comparison of the activations of the last ReLU by class in CU-ECG. 
Figure 17 shows a comparison of the highest accuracies between transfer and nontransfer learning on PTB-ECG. Figure 18 shows a comparison of the highest accuracies between transfer and nontransfer learning on CU-ECG. The accuracies were quite different among models trained with transfer, but the accuracies were slightly different among models trained without transfer. Models trained with transfer were optimized for previous training data. So, the large difference of accuracy between transfer and nontransfer means that the model is sensitive to training data. The differences of AlexNet, googleNet, and ResNet were 11.53, 2.32, and 0.15\%, respectively, on PTB-ECG, and the differences of AlexNet, googleNet, and ResNet were 37.05, 6.37, and 0.99\%, respectively, on CU-ECG. The ResNet has $11.38 \%$ and $2.17 \%$ lower differences between transfer and nontransfer, respectively, than AlexNet and googleNet on PTB-ECG. The ResNet has $36.06 \%$ and $5.38 \%$ lower differences between transfer and nontransfer, respectively, than AlexNet and googleNet on CU-ECG. Table 11 indicates comparison of computational time. The training times were measured during one epoch when the training method, minibatch size, and transferred units were Adam, 64, and X, and the test times were measurements on one signal processing.

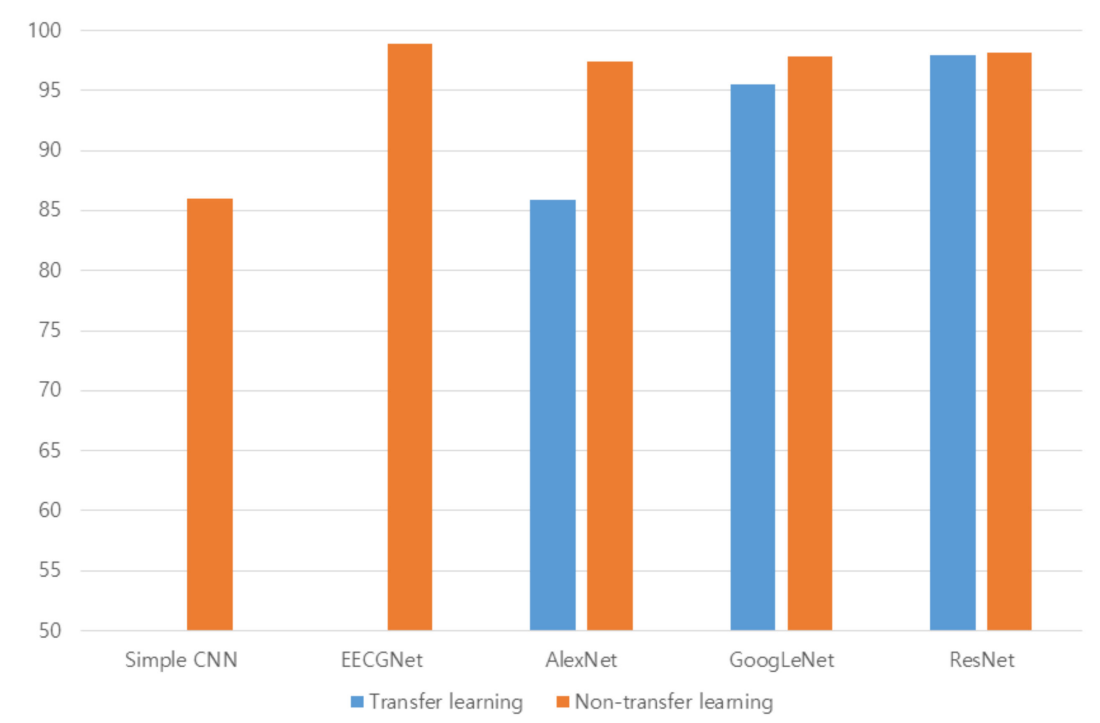

Figure 17. Comparison of the highest accuracies between transfer and nontransfer learning on PTB-ECG.

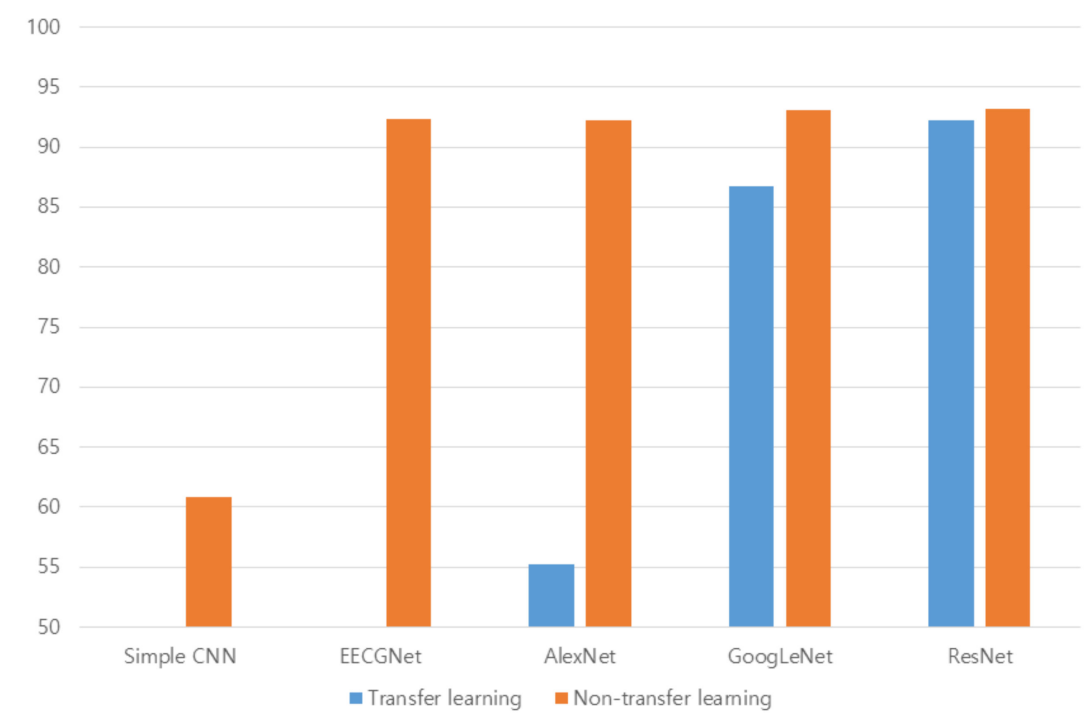

Figure 18. Comparison of the highest accuracies between transfer and nontransfer learning on CU-ECG. 
Table 11. Comparison of computational time.

\begin{tabular}{cccc}
\hline \multirow{2}{*}{ Model } & \multicolumn{2}{c}{ Training Time } & \multirow{2}{*}{ Test Time } \\
\cline { 2 - 3 } & PTB-ECG & CU-ECG & \\
\hline Simple CNN & $1 \mathrm{~m} 15 \mathrm{~s}$ & $1 \mathrm{~m} \mathrm{33} \mathrm{s}$ & $0.000423 \mathrm{~s}$ \\
AlexNet & $3 \mathrm{~m} \mathrm{38} \mathrm{s}$ & $4 \mathrm{~m} 26 \mathrm{~s}$ & $0.001809 \mathrm{~s}$ \\
GoogLeNet & $9 \mathrm{~m} 11 \mathrm{~s}$ & $11 \mathrm{~m} 37 \mathrm{~s}$ & $0.004704 \mathrm{~s}$ \\
ResNet & $77 \mathrm{~m} 28 \mathrm{~s}$ & $92 \mathrm{~m} \mathrm{36} \mathrm{s}$ & $0.012437 \mathrm{~s}$ \\
EECGNet & $31 \mathrm{~m} 53 \mathrm{~s}$ & $36 \mathrm{~m} 1 \mathrm{~s}$ & $0.295800 \mathrm{~s}$ \\
\hline
\end{tabular}

To measure performance of verification, the data is reconstructed into two classes of one-against-all. To balance the two sample sets in the case of PTB-ECG, only 61 people among 211 people were used because a person has 60 data. The first person is positive and the people from 2 to 61 are negative. The negative samples are constructed by taking one sample per person. The models are trained with training method, minibatch size, and transferred units as Adam, 60, and X. Only ResNet was 10 as minibatch size. $\mathrm{K}, \mathrm{L}, \mathrm{h}$, and $\mathrm{R}$ were 8, 8, 8, and 0.5 in EECGNet, respectively. The performances are measured on test dataset. Our research focused on individual identification. Table 12 shows verification performance on PTB-ECG. Figure 19 describes comparison of the ROC(Receiver Operating Characteristic) curves on PTB-ECG.

Table 12. Verification performance on PTB-ECG.

\begin{tabular}{ccccccc}
\hline Model & Accuracy (\%) & Sensitivity & Specificity & FPR & FNR & EER (\%) \\
\hline Simple CNN & 87.50 & 0.95 & 0.80 & 0.20 & 0.05 & 13.33 \\
AlexNet & 92.50 & 0.85 & 1.00 & 0.00 & 0.15 & 0.00 \\
GoogLeNet & 100.00 & 1.00 & 1.00 & 0.00 & 0.00 & 0.00 \\
ResNet & 99.17 & 1.00 & 0.9833 & 0.02 & 0.00 & 0.00 \\
EECGNet & 100.00 & 1.00 & 1.00 & 0.00 & 0.00 & 0.00 \\
\hline
\end{tabular}

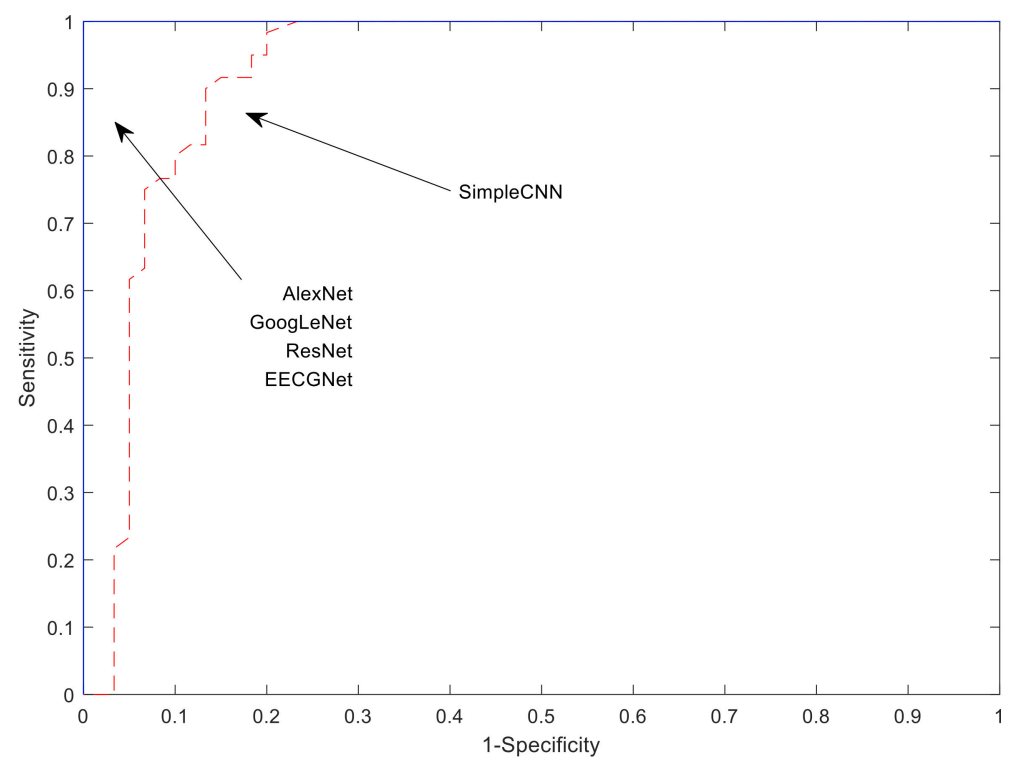

Figure 19. Comparison of the ROC (Receiver Operating Characteristic) curves on PTB-ECG.

\section{Conclusions}

We performed comparative analysis of deep models in biometrics using scalogram of electrocardiogram. Since biometrics using ECG signals are sensitive to noise, studies have been conducted by transforming signals into the frequency domain that is efficient for analyzing noisy signals. By transforming the signal from the time domain to the frequency domain using the 
wavelet, the 1-D signal becomes a 2-D matrix, and it could be analyzed at multiresolution. However, this process makes for a morphologically complex signal analysis. This means that existing simple classifiers could be poor in performance. We investigate the possibility of using the scalogram of electrocardiogram as input to CNN of deep learning, which exhibits optimal performance for the classification of morphological imagery. AlexNet, GoogLeNet, and ResNet are considered deep models of the convolutional neural network in this paper. The experiments are performed on two databases for performance evaluation. The ResNet was $0.73 \%-0.27 \%$ higher than AlexNet or googleNet on PTB-ECG—and the ResNet was $0.94 \%-0.12 \%$ higher than AlexNet or GoogleNet on CU-ECG. The ResNet had $11.38 \%$ and $2.17 \%$ lower differences between transfer and nontransfer, respectively, than AlexNet and googleNet on PTB-ECG. The ResNet had 36.06\% and 5.38\% lower differences between transfer and nontransfer, respectively, than AlexNet and googleNet on CU-ECG. For further research, we will study less influenceable ECG representation to noise.

Author Contributions: Conceptualization, K.-C.K. and Y.-H.B.; Methodology, K.-C.K. and Y.-H.B.; Software, K.-C.K. and Y.-H.B.; Validation, K.-C.K. and Y.-H.B.; Formal Analysis, K.-C.K. and Y.-H.B.; Investigation, K.-C.K. and Y.-H.B.; Resources, K.-C.K.; Data Curation, K.-C.K. and S.-B.P.; Writing-Original Draft Preparation, Y.-H.B.; Writing-Review and Editing, K.-C.K.; Visualization, K.-C.K. and Y.-H.B.; Supervision, K.-C.K.; Project Administration, S.-B.P.; Funding Acquisition, S.-B.P.

Funding: This research was supported by the Basic Science Research Program through the National Research Foundation of Korea (NRF) funded by the Ministry of Education (No. 2017R1A6A1A03015496) (No.2018R1D1A1B07044907).

Conflicts of Interest: The authors declare no conflicts of interest.

\section{References}

1. Wang, H.; Hu, J.; Deng, W. Compressing fisher vector for robust face recognition. IEEE Access 2017, 5, 23157-23165. [CrossRef]

2. Jain, A.K.; Arora, S.S.; Cao, K.; Best-Rowden, L.; Bhatnagar, A. Fingerprint recognition of young children. IEEE Trans. Inf. Forensics Secur. 2017, 12, 1505-1514. [CrossRef]

3. Nguyen, B.P.; Tay, W.L.; Chui, C.K. Robust biometric recognition from palm depth images for gloved hands. IEEE Trans. Human-Machi. Syst. 2015, 45, 799-804. [CrossRef]

4. Zhang, Y.; Juhola, M. On biometrics with eye movements. IEEE J. Biomed. Health Inform. 2017, 21, 1360-1366. [CrossRef] [PubMed]

5. Pokhriyal, N.; Tayal, K.; Nwogu, I.; Govindaraju, V. Cognitive-biometric recognition from language usage: A feasibility study. IEEE Trans. Inf. Forensics Secur. 2017, 12, 134-143. [CrossRef]

6. Boles, W.W. A security system based on human iris identification using wavelet transform. In Proceedings of the First International Conference on Conventional and Knowledge based Intelligent Electronics Systems, Adelaide, SA, Australia, 21-23 May 1997; pp. 533-541.

7. $\mathrm{Xu}, \mathrm{W}$.; Lee, E.J. A Hybrid method based on dynamic compensatory fuzzy neural network algorithm for face recognition. Int. J. Control Autom. Syst. 2014, 12, 688-696. [CrossRef]

8. Jang, Y.K.; Kang, B.J.; Kang, R.P. A novel portable iris recognition system and usability evaluation. Int. J. Control Autom. Syst. 2010, 8, 91-98. [CrossRef]

9. Hong, S.J.; Lee, H.S.; Tho, K.A.; Kim, E.T. Gait recognition using multi-bipolarized contour vector. Int. J. Control Autom. Syst. 2009, 7, 799-808. [CrossRef]

10. Kim, M.J.; Kim, W.Y.; Paik, J.K. Optimum geometric transformation and bipartite graph-based approach to sweat pore matching for biometric identification. Symmetry 2018, 10, 175. [CrossRef]

11. Yang, J.; Sun, W.; Liu, N.; Chen, Y.; Wang, Y.; Han, S. A novel multimodal biometrics recognition model based on stacked ELM and CCA methods. Symmetry 2018, 10, 96. [CrossRef]

12. Zhang, L.; Cheng, Z.; Shen, Y.; Wang, D. Palmprint and palmvein recognition based on DCNN and a new large-scale contactless palmvein dataset. Symmetry 2018, 10, 78. [CrossRef]

13. Korshunov, P.; Marcel, S. Impact of score fusion on voice biometrics and presentation attack detection in cross-database evaluations. IEEE J. Sel. Top. Signal Process. 2017, 11, 695-705. [CrossRef]

14. Tolosana, R.; Vera-Rodriguez, R.; Fierrez, J.; Ortega-Garcia, J. Exploring recurrent neural networks for on-line handwritten signature biometrics. IEEE Access 2018, 6, 5128-5138. [CrossRef] 
15. Byeon, Y.H.; Lee, J.N.; Pan, S.B.; Kwak, K.C. Multilinear EigenECGs and FisherECGs for individual identification from information obtained by ad electrocardiogram sensor. Symmetry 2018, 10, 487. [CrossRef]

16. Gahi, Y.; Lamrani, M.; Zoglat, A.; Guennoun, M.; Kapralos, B.; El-Khatib, K. Biometric identification system based on electrocardiogram data. In Proceedings of the New Technologies, Mobility and Security, Tangier, Morocco, 5-7 November 2008; pp. 1-4.

17. Kim, J.J.; Lee, S.M.; Ryu, G.S.; Lee, J.H.; Park, K.H. Hierarchical authentication algorithm using curvature based fiducial point extraction of ECG signals. J. Korea Multimedia Soc. 2017, 20, 465-473. [CrossRef]

18. Kim, J.K.; Lee, K.B.; Hong, S.G. ECG-based biometric authentication using random forest. J. Inst. Electr. Inf. Eng. 2017, 54, 100-105.

19. Lim, C.S. A study on the analysis of technology and service issues for wearable devices and future development direction. J. Korea Inst. Next Gener. Comput. 2017, 13, 81-89.

20. Li, T.; Zhou, M. ECG classification using wavelet packet entropy and random forests. Entropy 2016, 18, 285. [CrossRef]

21. Khorrami, H.; Moavenian, M. A comparative study of DWT, CWT and DCT transformation in ECG arrhythmias classification. Expert Syst. Appl. 2010, 37, 5751-5757. [CrossRef]

22. Song, M.H.; Lee, J.; Cho, S.P.; Lee, K.J.; Yoo, S.K. Support vector machine based arrhythmia classification using reduced features. Int. J. Control Autom. Syst. 2005, 3, 571-579.

23. Yu, S.N.; Chen, Y.H. Electrocardiogram beat classification based on wavelet transformation and probabilistic neural network. Pattern Recognit. Lett. 2007, 28, 1142-1150. [CrossRef]

24. Ye, C.; Coimbra, M.T.; Kumar, B. Arrhythmia detection and classification using morphological and dynamic features of ECG signals. In Proceedings of the Annual International Conference of the IEEE Engineering in Medicine and Biology Society, Buenos Aires, Argentina, 31 August-4 September 2010; pp. 1918-1921.

25. Soualhi, K.; Oueslati, A.E.; Ellouze, N. ECG image representation of normal sinus rhythm. In Proceedings of the International Conference on Advanced Technologies for Signal and Image Processing, Sousse, Tunisia, 17-19 March 2014; pp. 225-230.

26. Zhai, X.; Tin, C. Automated ECG classification using dual heartbeat coupling based on convolutional neural network. IEEE Access 2018, 6, 27465-27472. [CrossRef]

27. Pak, M.S.; Kim, S.H. A review of deep learning in image recognition. In Proceedings of the International Conference on Computer Applications and Information Processing Technology, Kuta Bali, Indonesia, 8-10 August 2017; pp. 1-3.

28. Narayan, S.; Tagliarini, G.A.; Page, E.W. Enhancing MLP networks using a distributed data representation. IEEE Syst. Man Cybern. Part B 1996, 26, 143-149. [CrossRef] [PubMed]

29. Chang, C.H. Deep and shallow architecture of multilayer neural networks. IEEE Neural Netw. Learn. Syst. 2015, 26, 2477-2486. [CrossRef] [PubMed]

30. Callet, P.L.; Viard-Gaudin, C.; Barba, D. A convolutional neural network approach for objective video quality assessment. IEEE Neural Netw. 2006, 17, 1316-1327. [CrossRef] [PubMed]

31. Mou, L.; Ghamisi, P.; Zhu, X.X. Deep recurrent neural networks for hyperspectral image classification. IEEE Trans. Geosci. Remote Sens. 2017, 55, 3639-3655. [CrossRef]

32. Diao, W.; Sun, X.; Zheng, X.; Dou, F.; Wang, H.; Fu, K. Efficient saliency-based object detection in remote sensing images using deep belief networks. IEEE Geosci. Remote Sens. Lett. 2016, 13, 137-141. [CrossRef]

33. Sasaki, H.; Horiuchi, T.; Kato, S. A study on vision-based mobile robot learning by deep Q-network. In Proceedings of the Annual Conference of Society of Instrument Control Engineers, Kanazawa, Japan, 19-22 September 2017; pp. 799-804.

34. Hou, J.C.; Wang, S.S.; Lai, Y.H.; Tsao, Y.; Chang, H.W.; Wang, H.M. Audio-visual speech enhancement using multimodal deep convolutional neural networks. IEEE Emerg. Top. Comput. Intell. 2018, 2, 117-128. [CrossRef]

35. Sutskever, A.I.; Hinton, G.E. ImageNet classification with deep convolutional neural networks. In Proceedings of the International Conference on Neural Information Processing Systems, Lake Tahoe, NV, USA, 3-6 December 2012; pp. 1097-1105.

36. Szegedy, C.; Liu, W.; Jia, Y.; Sermanet, P.; Reed, S.; Anguelov, D.; Erhan, D.; Vanhoucke, V.; Rabinovich, A. Going deeper with convolutions. In Proceedings of the IEEE Conference on Computer Vision and Pattern Recognition, Boston, MA, USA, 7-12 June 2015; pp. 1-9. 
37. He, K.; Zhang, X.; Ren, S.; Sun, J. Deep residual learning for image recognition. In Proceedings of the IEEE Conference on Computer Vision and Pattern Recognition, Las Vegas, NV, USA, 27-30 June 2016; pp. 770-778.

38. Shao, L.; Zhu, F.; Li, X. Transfer learning for visual categorization: A survey. IEEE Neural Netw. Learn. Syst. 2015, 26, 1019-1034. [CrossRef]

39. Lecun, Y.; Bottou, L.; Bengio, Y.; Haffner, P. Gradient-based learning applied to document recognition. Proc. IEEE 1998, 86, 2278-2324. [CrossRef]

40. Simonyan, K.; Zisserman, A. Very deep convolutional neural networks for large-scale image recognition. In Proceedings of the International Conference on Learning Representations, San Diego, CA, USA, 7-9 May 2015; pp. 1-14.

41. Israel, S.A.; Irvine, J.M.; Cheng, A.; Wiederhold, M.D.; Wiederhold, B.K. ECG to identify individuals. Pattern Recognit. 2005, 38, 133-142. [CrossRef]

42. Wang, Y.; Agrafioti, F.; Hatzinakos, D.; Plataniotis, K.N. Analysis of human electrocardiogram for biometric recognition. EURASIP J. Adv. Signal Process. 2007, 2008, 1-11. [CrossRef]

43. Seo, J.I.; Park, E.K.; Jang, J.Y. Comparison of ERG denoising performance according to mother function of wavelet transforms. J. Korean Clin. Health Sci. 2016, 4, 756-761. [CrossRef]

44. Olhede, S.C.; Walden, A.T. Generalized morse wavelets. IEEE Trans. Signal Process. 2002, 50, $2661-2670$. [CrossRef]

45. Lilly, J.M.; Olhede, S.C. Higher-order properties of analytic wavelets. IEEE Trans. Signal Process. 2009, 57, 146-160. [CrossRef]

46. Lilly, J.M.; Olhede, S.C. On the analytic wavelet transform. IEEE Trans. Inf. Theory 2010, 56, 4135-4156. [CrossRef]

47. Lilly, J.M.; Olhede, S.C. Generalized Morse wavelets as a superfamily of analytic wavelets. IEEE Trans. Signal Process. 2012, 60, 6036-6041. [CrossRef]

48. jLab: A Data Analysis Package for Matlab. Available online: http://www.jmlilly.net/jmlsoft.html (accessed on 20 November 2018).

49. Morse Wavelets. Available online: https://in.mathworks.com/help/wavelet/ug/morse-wavelets.html\# bvgfke1 (accessed on 15 November 2018).

50. Choi, H.S.; Lee, B.H.; Yoon, S.R. Biometric authentication using noisy electrocardiograms acquired by mobile sensors. IEEE Access 2016, 4, 1266-1273. [CrossRef]

51. Goldberger, A.L.; Amaral, L.N.; Glass, L.; Hausdorff, J.M.; Ivanov, P.C.; Mark, R.G.; Mietus, J.E.; Moody, G.B.; Peng, C.K.; Stanley, H.E. PhysioBank, PhysioToolkit, and PhysioNet: Components of a new research resource for complex physiologic signals. Circulation 2000, 101, e215-e220. [CrossRef] [PubMed]

52. Wubbeler, G.; Stavridis, M.; Kreiseler, D.; Bousseljot, R.D.; Elster, C. Verification of humans using the electrocardiogram. Pattern Recognit. Lett. 2007, 28, 1172-1175. [CrossRef]

53. An Overview of Gradient Descent Optimization Algorithms. Available online: https://arxiv.org/abs/1609. 04747 (accessed on 10 November 2018).

54. Lee, J.N.; Byeon, Y.H.; Pan, S.B.; Kwak, K.C. An EigenECG network approach based on PCANet for personal identification from ECG signal. Sensors 2018, 18, 4024. [CrossRef] [PubMed]

(C) 2019 by the authors. Licensee MDPI, Basel, Switzerland. This article is an open access article distributed under the terms and conditions of the Creative Commons Attribution (CC BY) license (http:// creativecommons.org/licenses/by/4.0/). 